Engineering and Computational Mechanics Volume 168 Issue EM1

Modelling of vortex flow controls at high drainage flow rates

Jarman, Butler, Tabor and Andoh
Proceedings of the Institution of Civil Engineers

Engineering and Computational Mechanics 168 March 2015 Issue EM1 Pages 17-34 http://dx.doi.org/10.1680/eacm.14.00006

Paper 1400006

Received 28/03/2014

Published online 19/12/2014

hydrodynamics

ICE Publishing: All rights reserved

\title{
Modelling of vortex flow controls at high drainage flow rates
}

Daniel Jarman MSc, PhD

Group Technology Manager, Hydro International, Clevedon, UK

David Butler MSC, DIC, PhD, CEng, CEnv, FICE, FICIWEM, FHEA

Senior Lecturer, College of Engineering, Maths and Physical Sciences,

University of Exeter, Exeter, UK
Gavin Tabor DPhil

Professor of Water Engineering, College of Engineering, Maths and Physical Sciences, University of Exeter, Exeter, UK

Robert Andoh MSC, DIC, PhD

Director of Innovations, Hydro International, Clevedon, UK

A number of vortex flow control (VFC) devices for urban drainage systems are investigated computationally at high flow rates, for which a confined vortex dominates the flow regime. A range of turbulence models, including both eddy viscosity and Reynolds stress closures, are compared with in-house experimental measurements of head loss and internal pressure measurements. Single-phase and multi-phase (free surface) calculations are also compared. Very good agreement with the experimental data was obtained when the swirl parameter of the device was below $3 \cdot 14$ for predictions made using the Reynolds stress closure formulations. For devices with swirl parameters above this value, the computational methodology was found to under-predict the head loss of the device. This was attributed to poor calibration of the turbulence model for swirling flow scenarios in which the pressure gradient and diffusive (turbulent) forces in the flow are comparable.

\section{Notation}

$A_{\mathrm{i}} \quad$ azimuthal inlet area

$C_{\mu} \quad$ model constant

$D_{\mathrm{c}}, D_{\mathrm{o}} \quad$ chamber, outlet diameters

$\mathbf{D}_{\mathrm{T}}, \mathbf{D}_{\mathrm{L}} \quad$ turbulent, molecular diffusion terms

E log-law wall constant

Eu Euler number

$P \quad$ stress production

$P_{\mathrm{k}} \quad$ turbulent production term

$\mathrm{R} \quad$ Reynolds stress

$\bar{S} \quad$ mean strain tensor

$S_{\mathrm{g}} \quad$ swirl parameter

$U_{\mathrm{w}} \quad$ wall velocity

$k$

$k_{\mathrm{w}}$

$l_{\mathrm{b}}$

$l_{\mathrm{m}}$

$\Delta \bar{p}$

$p$

$\bar{u}_{\mathrm{i}}, \bar{u}_{\mathrm{o}}$

$\bar{u}$

$y_{\mathrm{w}}$

$\alpha$

$\delta_{\mathrm{b}}$

$\varepsilon$

$\kappa$

$\mu$ turbulent kinetic energy

turbulent kinetic energy in the cell adjacent to the wall

circumference of VFC chamber

characteristic eddy scale

mean differential pressure across VFC

ensemble average pressure

mean velocity at inlet, outlet ports of VFC

ensemble average velocity

distance of first cell from wall

volume fraction

boundary layer height

turbulent dissipation

von Karman constant (0.41)

molecular viscosity

turbulent viscosity

pressure strain

fluid density

turbulent Schmidt number

$\begin{array}{ll}\tau & \text { mean viscous stress tensor } \\ \tau & \text { wall shear stress } \\ \omega & \text { specific dissipation rate }\end{array}$

\section{Introduction}

Vortex flow control (VFC) devices are used to regulate flow volumes and flow rates in urban drainage systems. For example, VFCs are often used to regulate the flow of water from an upper catchment region to a lower catchment region with the aim of balancing the flood risk or the potential socioeconomic cost of flooding between the upper and lower regions. VFCs are particularly advantageous compared with conventional flow regulators as they are passive, self-activating throttling devices with no mechanical components.

Previous researchers have recommended vortex valve geometries that maximise the head loss at a given flow rate (Brombach, 1972; King, 1985; Priestman, 1987; Wojtowicz and Kotowski, 2009; Zobel, 1936). However, maximising the head loss of the VFC is not always desirable as this can over-restrict the flow and increase the flood risk on the upstream catchment. Furthermore, VFCs exhibit a bi-stable throttling behaviour when positioned so that the discharge direction is horizontal. It is hypothesised that the bi-stable hydraulic characteristic can be tailored to deliver behaviour approaching a constant discharge by modifying the VFC geometry. A constant discharge condition is beneficial, as the pass-forward flow rate can be limited to a safe or permissible value and the average flow rate over a given rainfall period is maximised. Maximising the average flow rate reduces the volume of rainfall retained on the upstream region of the catchment and can help reduce flood mitigation infrastructure costs (Andoh and Declerck, 2005; Newton et al., 2013). 
The authors' research has been directed towards determining how each of the bi-stable behaviours is affected by the device geometry in order to determine rules for the design of the appropriate VFC for a given scenario. Previous work by the authors (Queguineur et al., 2013) examined the computational modelling of flow through a VFC at low flow rates where vortexing was not occurring. In this regime, the flow through the outlet orifice was found to be similar to flow over a thin plate weir. The current paper examines VFC behaviour at higher flow rates, when a confined vortex is present. The behaviour of VFCs for drainage systems operating in the vortex flow regime has been investigated before, but not commonly using computational fluid dynamics (CFD) (Deamer, 1988; Green, 1988; Parsian and Butler, 1993; Wojtowicz and Kotowski, 2009). The primary objective of the current work was to investigate the ability of a variety of Reynolds-averaged Navier-Stokes (RANS) based turbulence models in simulating turbulent swirling flow in a VFC. Turbulent losses within the vortex are a significant contribution to the throttling process, and so the proper behaviour of the turbulence model is critical to making predictions about the behaviour of these devices. The CFD predictions were compared with experimental data from a specially constructed test facility sited at Hydro International's offices in Clevedon, UK (Jarman, 2011; LeCornu and Faram, 2006).

\section{Operation of VFCs}

The geometries of VFC devices are generally defined by a cylindrical or conical frustum chamber with an inlet and outlet port. The outlet port of the chamber is positioned on the axis of the device. The inlet port is positioned on the azimuth of the chamber so that it imparts a swirling motion to the flow. The hydraulic behaviour of these devices is influenced by their geometry and orientation. When they are positioned so that the discharge direction is horizontal, these devices exhibit a bi-stable characteristic. This positioning causes air to be trapped within the unit above the outlet soffit level, which suppresses the vortex behaviour. For low velocities, gravitational effects are dominant and normal orifice-type flow patterns occur. As the flow rate and azimuthal velocity increase, air within the unit is expelled and the vortex motion becomes dominant. This results in additional inertial and turbulent losses across the VFC due to the vortex motion, further throttling the flow. The transition between these two bi-stable modes is not smooth, resulting in a complex hydraulic characteristic for VFCs.

A hydraulic characteristic for a VFC with a $100 \mathrm{~mm}$ dia. outlet in horizontal discharge orientation is shown by Figure 1. Where air entrainment is possible from downstream (i.e. a free-discharge condition), the transition phase is associated with the formation of an air core. This is analogous with a multi-phase vortex sink (bathplug vortex). This is due to an increase in hydraulic losses across the vortex, which causes the stagnation point to move from a downstream position onto the axis of the vortex.

The design of a flow control is primarily governed by the maxi-

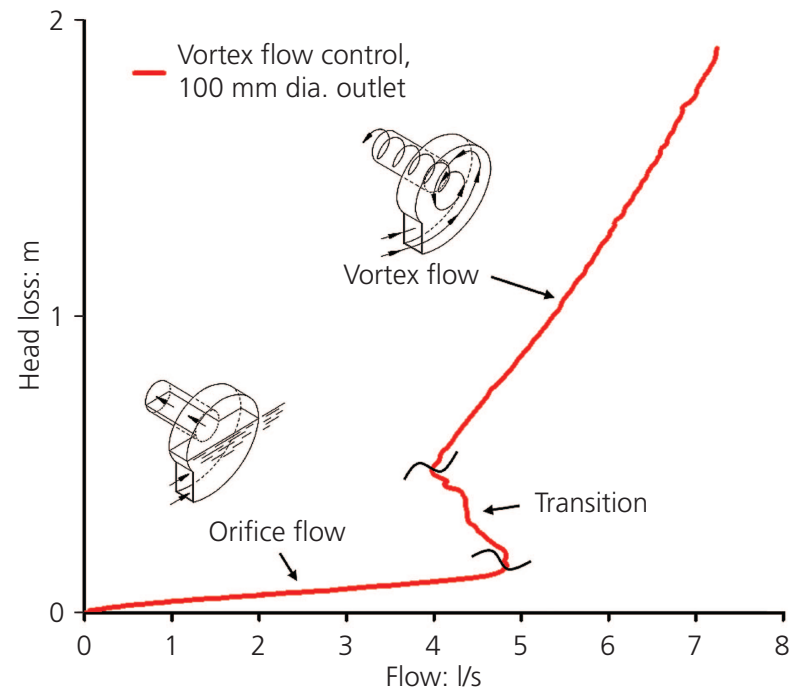

Figure 1. Hydraulic characteristics of a VFC with a $100 \mathrm{~mm}$ dia. outlet

mum head the device is expected to experience and the maximum permissible flow rate. For a VFC, the geometry should be manipulated so that the primary design point occurs during the vortexing phase operation. For this condition, the transition phase acts to increase the average flow rate over the VFC's operating head range, compared with a typical restrictive flow control device, such as an orifice (as shown in Figure 1). Minimising the difference between the average flow rate and the maximum design flow rate allows the VFC to approach a constant discharge condition.

\section{Computational methodology}

The CFD code adopted for this work was the open source code OpenFOAM (Weller et al., 1998). This has the ability to solve the RANS equations, the associated turbulence model, plus freesurface modelling by way of the volume of fluid (VoF) methodology using the finite-volume method on arbitrary unstructured polyhedral meshes. Properly, OpenFOAM is not a CFD code, but a C++ class library for writing CFD codes; the distribution comes with a number of pre-written applications, including several aimed at solving free-surface flow problems. Three pre-written solvers were used for the analysis in this work

- simpleFoam: a RANS solver for steady, incompressible, homogeneous, turbulent flows

nisoFoam: a RANS solver for unsteady, incompressible, homogeneous, turbulent flows

- interFoam: a RANS solver for unsteady, incompressible, immiscible, multi-phase, turbulent flows based on pisofoam, using the VoF methodology to represent the multi-phase system and free surface.

All of the solvers evaluate the transport equations in a segregated and sequential manner. Mass continuity, for the incompressible fluid system, is satisfied by the application of the simple 


\section{Offprint provided courtesy of www.icevirtuallibrary.com Author copy for personal use, not for distribution}

pressure-velocity coupling method in simpleFoam and the Piso method for the other unsteady solvers. In the interFoam solver, phase continuity is maintained by way of the MULES algorithm (http://www.opencfd.co.uk).

To establish a valid CFD modelling methodology for the VFCs, four factors were assessed to establish their effect on the accuracy of the predictions. These were mesh sensitivity, turbulence model behaviour, solution sensitivity to transient effects and the inclusion of the air phase to represent free-discharge conditions. Mesh sensitivity was investigated by varying the mean spatial discretisation length within the volume of the VFC chamber. The ability of the turbulence models to make accurate predictions was assessed by comparing the flow predictions from the five turbulence models discussed in Section 3.3 with the experimental findings. To examine the sensitivity of the solution to transient flow features, CFD predictions were also performed in which the temporal derivatives were neglected. This was considered an important aspect of the modelling methodology for investigation, as it has the potential to reduce significantly the computation cost for modelling swirling flows such as those present in VFCs. The accuracy of the multi-phase modelling approach was evaluated by comparing the predictions with the experimental findings for surcharged (singlephase) and free-discharge (multi-phase) conditions.

Only the vortexing phase of operation was simulated using CFD. Orifice-type flow was previously studied in detail by Queguineur et al. (2013). Simulating the behaviour of the transition phase was considered beyond the scope of this investigation as the transition is unsteady and exhibits a high degree of hydraulic variability over a relatively small flow rate range. This makes it computationally expensive to investigate this phase of operation for a single VFC and impractical for studying a large range of geometries. The modelling approach employed to investigate the vortexing behaviour of VFCs was to simulate discrete flow rates for a number of geometries and average the resultant quasi-steady flow parameters.

\subsection{VFC geometry}

A cylindrical-type, square-edge VFC geometry was selected for this research as this is the most common VFC geometry installed in stormwater drainage systems. The cylindrical-type VFC geometries studied in this work are defined by a cylindrical chamber positioned so that the axis of the chamber is horizontal and aligned with the discharge flow direction. In one quadrant of the chamber the radial cross-section of the chamber is extruded perpendicularly to the section for a distance equal to the chamber radius. This results in a square corner in one of the chamber quadrants and permits a square or rectangular inlet approximating an azimuthal entrance. The upstream face of the chamber is closed, whereas the downstream face possesses a circular outlet port, which is concentric with the cylindrical chamber.

The modelled computational domain consisted of the inlet pipe, the VFC test chamber, the VFC and an outlet pipe. The geometries of the inlet pipe, the VFC test chamber and the outlet pipe were chosen to match the experimental hydraulic test facility at the laboratory in Clevedon. Figure 2 shows a dimetric projection of the geometry for a VFC mounted in the test tank.

The vortexing behaviour is governed by the amount of swirl induced by the VFC. Swirl can be quantified by the dimensionless ratio of the axial flux of the swirl momentum divided by the product of the axial flux of the axial momentum and swirl radius. The amount of swirl induced by a VFC geometry can be inferred by a swirl parameter $S_{\mathrm{g}}$, based purely on the device geometry (Gupta et al., 1984)

$$
\text { 1. } S_{\mathrm{g}}=\frac{\pi D_{\mathrm{c}} D_{\mathrm{o}}}{4 A_{\mathrm{i}}}
$$

where $D_{\mathrm{c}}$ and $D_{\mathrm{o}}$ are the chamber and outlet diameters, respectively, and $A_{\mathrm{i}}$ is the azimuthal inlet area. Increasing levels of swirl tend to increase the inertial and turbulent losses through the VFC.

The Euler number is the ratio of potential to kinetic energy

$$
\text { 2. } \mathrm{Eu}=\frac{2 \Delta \bar{p}}{\rho \bar{u}_{\mathrm{o}}^{2}}
$$

where $\Delta \bar{p}$ is the mean differential pressure across the VFC and $\bar{u}_{\mathrm{o}}$ is the mean velocity at the outlet port of the VFC. By considering the relative pressure loss in the form of the Euler number, both the flow rate and head loss parameters are evaluated and direct comparisons between the various VFC geometries investigated can be made. For the experimental assessments, the pressure differential was measured by means of upstream and downstream manometers; where a free-discharge condition was applied the

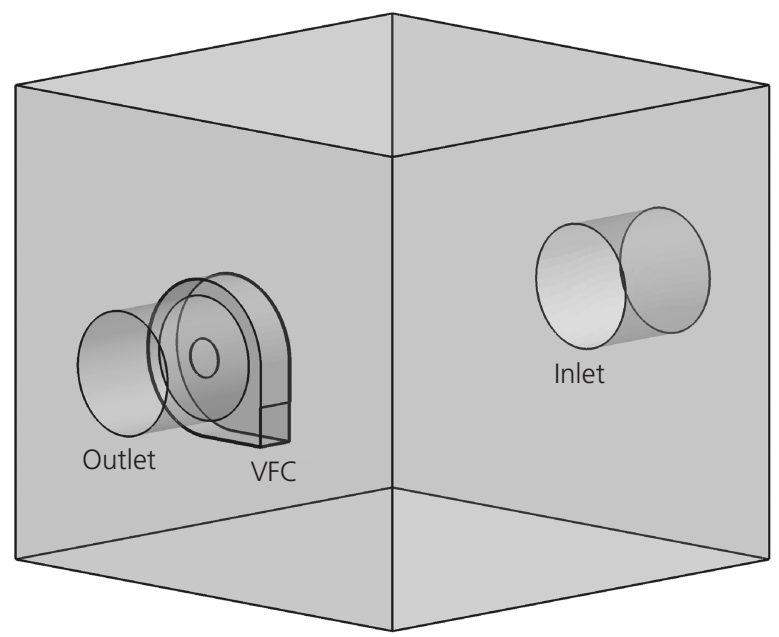

Figure 2. Simulated geometry for a VFC with a $100 \mathrm{~mm}$ dia. outlet and swirl parameter of $3 \cdot 14$ 
downstream pressure was assumed to be atmospheric. The simulated head loss across the VFCs was measured between the volume centroid of the upstream tank and outlet boundary, which made it comparable to the experimental physical assessment procedure.

The range of geometries and flow rates simulated and evaluated experimentally to validate the CFD modelling procedure is shown in Table 1. The geometries are listed against their associated swirl parameter. All scenarios listed in Table 1 were simulated using both the steady single-phase and unsteady multi-phase methods. The simulated flow rates were selected to correspond with the flow rate ranges assessed during the experimental testing.

To validate further the CFD modelling approach, internal pressure measurements were recorded during the experimental evaluations of the VFC unit with a swirl parameter of $3 \cdot 14$. The internal VFC pressure distributions were measured at 19 equidistant locations across the internal upstream face of the VFC along a horizontal plane that passed through the axis of the outlet port, as shown in Figure 3. Experimental errors in the measurements amounted to $\pm 1 \cdot 36 \%$ ( $95 \%$ confidence level). Readings could only be obtained for surcharged conditions as the free-discharge condition was found to cause air locks in the manometers and result in erroneous measurements.

\subsection{Computational mesh}

All of the simulated domains were discretised using an unstructured, hexahedral mesh created using the ANSYS Gambit meshing software. This type of mesh was chosen as it allows suitable control of the discretisation length in the near-wall region, ensuring satisfactory behaviour of the wall function relationship. To assess the sensitivity of the solution to the spatial discretisation, the mean cell length was varied by the required number of control volumes across the diameter of the VFC chamber. This is more clearly demonstrated in Figure 4, which shows a VFC with a $100 \mathrm{~mm}$ dia. outlet and a geometric swirl parameter of $3 \cdot 14$ with 80 control volume divisions across the VFC chamber diameter. Mean discretisation lengths equivalent to 40, 80, 110 and 140 divisions of the VFC were used to assess the solution sensitivity to spatial discretisation. This resulted in the number of control volumes within the chamber of the VFC varying between approximately 100000 and 4500000 . As wall functions were applied, the spatial discretisation length normal to the walls needed to be similar to the boundary layer height. The boundary

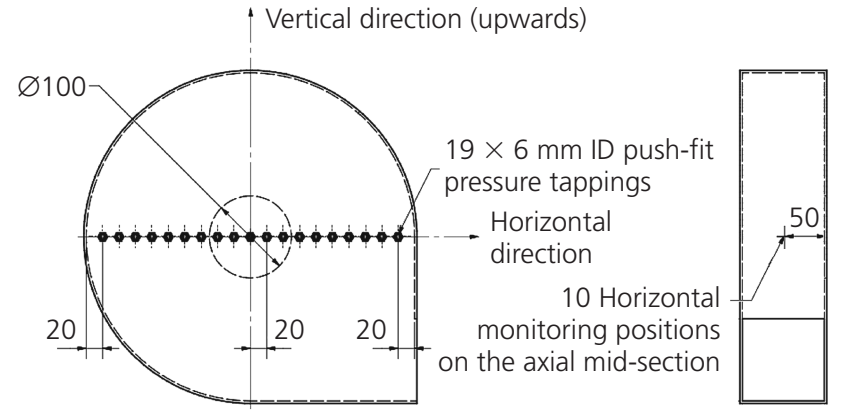

Figure 3. Illustration of pressure and velocity monitoring positions for a VFC with a $100 \mathrm{~mm}$ dia. outlet and a swirl parameter of $3 \cdot 14$ (dimensions in $\mathrm{mm}$ )

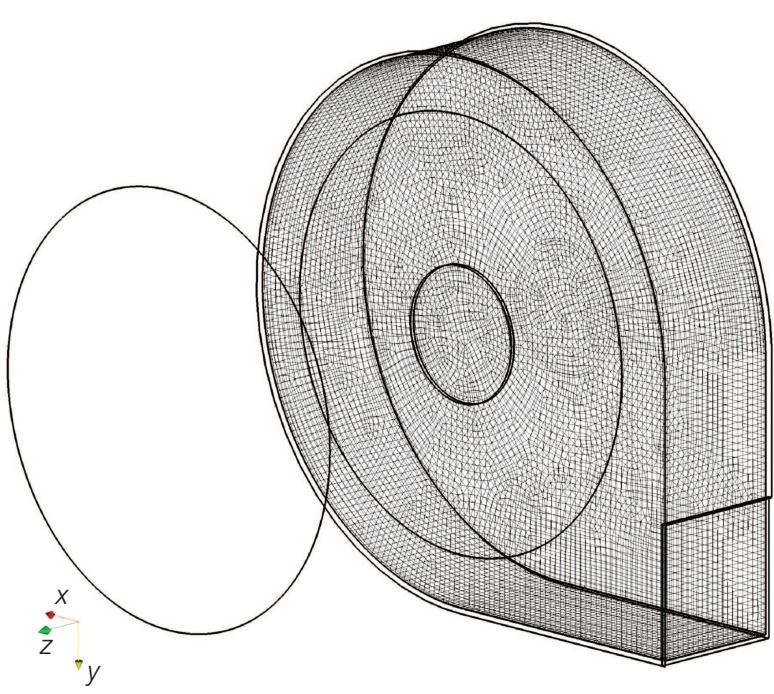

Figure 4. Spatial discretisation of a VFC with a $100 \mathrm{~mm}$ dia. outlet and a swirl parameter of $3 \cdot 14$ using one 1/80th of the chamber length as the mean control volume length

layer was assumed to be turbulent and its height was predicted by way of the relationship (Schlichting et al., 2004)

3. $\delta_{\mathrm{b}}=0 \cdot 37 l_{\mathrm{b}}\left(\frac{\rho \bar{u}_{\mathrm{i}} l_{\mathrm{b}}}{\mu}\right)^{-1 / 5}$

Swirl parameter Outlet diameter: $\mathrm{mm} \quad$ Chamber diameter: $\mathrm{mm} \quad$ Inlet area: $\mathrm{mm}^{2} \quad$ Axial length: $\mathrm{mm}$ Simulated flow rate: $1 / \mathrm{s}$

\begin{tabular}{llllll}
\hline $1 \cdot 57$ & 200 & 10000 & 100 & $4,6,7,10,13,16$ \\
$3 \cdot 14$ & 100 & 400 & 10000 & 100 & $4,5,6,7,8,9,10,11$ \\
$4 \cdot 71$ & 100 & 600 & 10000 & 100 & $4,5,6,7,8$ \\
$6 \cdot 28$ & 100 & 800 & 10000 & 100 & $4,5,6,7,8$
\end{tabular}

Table 1. Simulated VFC geometries flow conditions 
where $\delta_{\mathrm{b}}$ is the boundary layer height, $l_{\mathrm{b}}$ is the length of the circumference of the VFC chamber and $\bar{u}_{\mathrm{i}}$ is the mean inlet velocity. As the circulation remains relatively constant in the outer vortex tail region, the velocity of the flow increases as the flow approaches the outlet. In order to ensure the validity of the wall function relationship (defined in Section 3.3.1) the mesh was refined or unrefined, as required. Analysis of spatial discretisation resolutions above 140 divisions of the chamber diameter was not considered appropriate as this resulted in a mean discretisation length that was smaller than the boundary layer height in some cases. Under these conditions, the wall functions and turbulence models selected for this study would not be well suited and a low Reynolds number turbulence modelling approach would be more appropriate.

\subsection{Turbulence models}

Vortex flow controls of practical interest to urban drainage systems operate with a Reynolds number in the region of $10^{5}$. This indicates that turbulent effects are likely to have an influence on the hydraulic behaviour of these devices. Applying an ensemble average to the Navier-Stokes equations for incompressible flow produces the RANS equations

\section{4. $\quad \nabla \cdot \overline{\mathbf{u}}=0$}

diffusion, respectively, $\mathbf{P}$ represents stress production, $\Pi$ is the pressure strain and $\varepsilon$ is the turbulent dissipation. The terms for molecular diffusion and stress production may be evaluated directly as they only contain quantities of mean velocity or the Reynolds stresses. The other terms must be modelled in order to close the RANS system of equations. This is the basis of differential second-moment turbulence closure (DSM) models.

In the second family of turbulence models the Boussinesq approximation is applied, where it is assumed that the turbulent stresses vary linearly with the mean fluid strain and behave in a similar fashion to the molecular stresses (as for an incompressible Newtonian fluid). This produces a linear relationship between the Reynolds stresses and the mean velocity gradients

$$
\text { 7. } \quad \mathbf{R}=\frac{2}{3} \rho \mathbf{I} k-2 \mu_{\mathrm{t}} \overline{\mathbb{S}}
$$

where $k$ represents the turbulent kinetic energy per unit mass and $\mu_{\mathrm{t}}$ is known as the eddy viscosity (or turbulent viscosity). This allows Equation 6 to be simplified to a transport equation for $k$, eliminating many of the unknown quantities

$$
\text { 8. } \frac{\partial(\rho k)}{\partial t}+\nabla \cdot(\rho k \overline{\mathbf{u}})=P_{k}-\rho \varepsilon+\nabla \cdot\left[\left(\mu+\frac{\mu_{\mathrm{t}}}{\sigma_{k}}\right) \nabla k\right]
$$

where $P_{k}$ is the production term, and the term $\mu_{\mathrm{t}} / \sigma_{k}$ represents the turbulent diffusivity, where $\sigma_{k}$ is the turbulent Schmidt number. Due to the presence of the viscous-like quantity $\mu_{\mathrm{t}}$, turbulence models based on the Boussinesq approximation are often referred to as eddy viscosity models (EVMs).

A common requirement of both the DSM and EVM approaches is a closure model for turbulent dissipation $(\varepsilon)$, which can be constructed through dimensional and physical reasoning.

is the mean viscous stress tensor, $\overline{\mathbf{u}}$ is the ensemble average velocity and $\mathbf{R}$ is referred to as the Reynolds stress $\left(\overline{\mathbf{u}}^{\prime} \overline{\mathbf{u}}^{\prime}\right)$, which represents the effect of the turbulent component of the flow on the mean flow. As this represents an additional six variables, the equation set is no longer closed and we have to introduce a mathematical model, known as the turbulence model, to relate this unknown quantity to the known averaged variables. From this starting point, two complete families of turbulence models may be derived. In one, a transport equation for the Reynolds stresses, the Reynolds stress transport (RST) equation, can be obtained by multiplying the Navier-Stokes momentum equation by the fluctuating components of the velocity field and averaging the product, giving the result

$$
\text { 6. } \frac{\partial(\mathbf{R})}{\partial t}+\nabla \cdot(\overline{\mathbf{u}} \mathbf{R})=\mathbf{D}_{\mathrm{T}}+\mathbf{D}_{\mathrm{L}}+\mathbf{P}+\Pi-\rho \varepsilon
$$

where the terms $\mathbf{D}_{\mathrm{T}}$ and $\mathbf{D}_{\mathrm{L}}$ represent turbulent and molecular
The solution of a separate transport equation for each component of the turbulent stress, as in DSM models, enables, in principle, accurate prediction of the turbulent stress field and its anisotropy. This is valuable in resolving the structure and orientation of the stress-bearing turbulent eddies that play a crucial role in turbulence dynamics in complex flows (e.g. in rotating and swirling flows), which can be a deficiency of EVMs. In turn, the representation of production and dissipation terms as scalar quantities in the EVM's results in the Reynolds stresses becoming isotropic, which is a significant simplification. However, DSMs do not always show superiority over two-equation EVMs. There are two primary reasons for this - the difficulties in establishing the behaviour of the additional terms in the DSM models and the additional numerical complexities associated with solving the six DSM stress transport equations (Launder and Sandham, 2002; Wilcox, 1994). EVMs are also attractive as they are roughly half as computationally demanding as DSM models. For this reason, 
three EVMs and two DSMs were evaluated to assess their suitability for simulating the behaviour of VFCs.

The EVM models investigated as part of this work were

- the standard $k-\varepsilon$ model (Jones and Launder, 1972; Launder and Sharma, 1974)

- the realisable $k-\varepsilon$ model (Shih et al., 1995)

- the $k-\omega$ shear stress transport (SST) model (Menter and Esch, 2001).

The standard $k-\varepsilon$ model is the benchmark two-equation EVM. The realisable $k-\varepsilon$ model has been shown to give superior results in regions of high, free-stream, strain rate over the standard $k-\varepsilon$ model in most situations (Shih et al., 1995). This is a result of imposing additional terms that limit the Reynolds stresses from becoming negative in regions of high strain rate (see Equation 7), which is possible for the standard $k-\varepsilon$ model of Launder and Sharma (1974). Along with satisfying the Schwarz inequality, this ensures the model is realisable (Launder and Sandham, 2002). This is an important criterion in preventing the model producing unphysical results and improves the response of the model in regions of varying strain (e.g. in rotating and swirling flows). Kolmogorov (1942) derived a transport equation for the specific dissipation rate $\omega$ rather than the absolute dissipation rate $\varepsilon$. Models of this type have been shown to be superior to the $k-\varepsilon$ approach for boundary layer dominated flows, but do not perform as well as the $k-\varepsilon$ models for free stream and wake conditions because the $k-\omega$ model can be overly sensitive to the turbulent boundary conditions for inlets (Hoffmann and Chiang, 2000). In an attempt to benefit from the best features of both dissipation treatments, Menter and Esch (2001) blended the $k-\varepsilon$ and $k-\omega$ methods to provide a more universal turbulence model, resulting in the $k-\omega$ SST model.

The two DSM models investigated were those proposed by Launder et al. (1975) and a closely related model proposed by Gibson and Launder (1978). The modelling approach taken by Launder et al. (1975) is to close the RST relationship (Equation 6) by examining each term individually. Only the contributions due to turbulent diffusion, turbulent dissipation rate and pressure strain production require modelling, as the other terms can be evaluated directly. The second-moment closure method proposed by Gibson and Launder (1978) differs only marginally from the model proposed by Launder et al. (1975) in that it includes pressure strain, wall echo and blockage terms. The aim of these terms is to capture the physics of the eddies stretching and splattering in the near-wall region, which has a tendency to increase eddy anisotropy. This differs from the other pressure strain terms, which act to decrease the stress anisotropy of the eddies.

\subsubsection{Near-wall behaviour}

Due to the no-slip condition imposed at a typical wall boundary, the strain rate in this region is large. Therefore, walls often present as a significant source of turbulent stress production. In order to resolve these gradients directly, spatial discretisation of sufficient resolution is required, which can be computationally expensive. A cheaper alternative is to apply an empirical wall function relationship in the near-wall region in order to enforce the required behaviour. This is most commonly performed by adjusting the near-wall viscosity to produce the expected nearwall shear stress $\left(\tau_{\mathrm{w}}\right)$ for turbulent conditions. The wall function is implemented by imposing the non-dimensional logarithmic velocity profile proposed by Launder and Spalding (1974)

9. $\quad u^{+}=\frac{U_{\mathrm{w}}}{\left(\tau_{\mathrm{w}} / \rho\right)^{1 / 2}}=\frac{1}{\kappa} \ln \left(E y^{+}\right)$

where $U_{\mathrm{w}}$ is the velocity parallel to the wall in the bounding cell, $\tau_{\mathrm{w}}$ is the wall shear stress, $\kappa$ is the von Karman constant $(0 \cdot 41)$ and $E$ is an empirically derived constant $(E=9.8$ for smooth walls) (Ferziger and Peric, 2002). This relationship requires that the wall distance must also be considered non-dimensionally

10. $y^{+}=\frac{\rho y_{\mathrm{w}}\left(\tau_{\mathrm{w}} / \rho\right)^{1 / 2}}{\mu}$

where $y_{\mathrm{w}}$ is the first cell spacing from the wall and $k_{\mathrm{w}}$ is the turbulence kinetic energy in the cell adjacent to the wall. Empirical studies of boundary layer development for linear flow along flat plates have shown that for $y^{+}<11.63$ the flow is laminar. For $11.63<y^{+}<300$, turbulent effects are present and the empirical relationship between Equations 9 and 10 is valid (Launder and Spalding, 1974; Versteeg and Malalasekera, 1995). Although for the VFCs the flow is swirling and many of the walls are not flat, this relationship was utilised to reduce computational requirements. This was considered a relatively common extrapolation of the relationship.

The wall function relationship allows the shear stress in the cell adjacent to the wall to be adjusted by specifying the effective viscosity $\left(\mu_{\mathrm{e}}=\mu+\mu_{\mathrm{t}}\right)$ according to Equations 11 and 12 , while assuming a zero gradient condition for the turbulent kinetic energy

$\mu_{\mathrm{e}}=$
$\begin{cases}\mu & y^{+}<11.63 \\ \frac{\mu y^{+}}{11.63+(1 / \kappa) \ln \left[1+\kappa\left(y^{+}-11.63\right)\right]} & y^{+}>11.63\end{cases}$ 
Values for $\varepsilon$ in the wall region are calculated using the resulting value of turbulent kinetic energy from $\varepsilon=C_{\mu} k^{2 / 3} / l_{\mathrm{m}}$, where $C_{\mu}$ is a proportionality constant and the mixing length is defined as $l_{\mathrm{m}}=\kappa y_{\mathrm{w}}$. The specific dissipation rate $(\omega)$ at the wall is defined by blending between the viscous sublayer and wall function behaviours

13.

$$
\omega_{\mathrm{w}}=\left[\left(\frac{6 \mu}{\rho 0 \cdot 075 y_{\mathrm{w}}^{2}}\right)^{2}+\left(\frac{k_{\mathrm{w}}^{0.5}}{0 \cdot 09^{0 \cdot 25} \kappa y_{\mathrm{w}}}\right)^{2}\right]^{1 / 2}
$$

This enforces appropriate behaviour at the wall without the need for fine spatial discretisation.

\subsection{The volume of fluid method}

Hirt and Nichols (1981) introduced the VoF method in order to solve flow problems with moving free surfaces. The evolution of the interface separating two different phases can be described using a discrete function whose value in each cell of the computational domain is equal to the volume fraction $\alpha$ defined as

- $\alpha=1 \rightarrow$ control volume is filled only with phase 1

- $\alpha=0 \rightarrow$ control volume is filled only with phase 2

- $0<\alpha<1 \rightarrow$ interface present in control volume.

As the volume fraction $\alpha$ is smoothly varying between 0 and 1 , the interface is never sharply defined, but occupies a volume around the region where a sharp interface should exist (Gopala and Wachen, 2007). The evolution of the volume fraction $\alpha$ is governed by the advection equation

14. $\frac{\partial \alpha}{\partial t}+\nabla \cdot(\alpha \overline{\mathbf{u}})=0$

It is necessary to solve Equation 14 in such a way as to generate a sharp interface. The discretisation of the advection term must limit numerical diffusion and oscillations at the phase interfaces. Jasak and Weller (1995) introduced a compression term into Equation 14, giving

15. $\frac{\partial \alpha}{\partial t}+\nabla \cdot(\alpha \overline{\mathbf{u}})+\nabla \cdot\left(\mathbf{u}^{r} \alpha(1-\alpha)\right)=0$

where $\mathbf{u}^{r}$ is a velocity field suitable for the compression of the interface. Due to the term $\alpha(1-\alpha)$, the compression term is active only at the interface where the volume fraction $\alpha$ varies between 0 and 1. The solution to the discretised form of Equation 15 is determined using the inter-gamma differencing scheme based on the donor-acceptor method and normalised variable diagram formulated by Leonard (1988). This scheme preserves the boundedness of $\alpha$ by introducing a certain amount of numerical diffusion while at the same time preserving a reasonably sharp resolution of the interface.

The fluid mixture properties are determined through linear interpolation between the properties of the fluid phases using the volume fraction $\alpha$

16. $\rho_{\mathrm{m}}=\alpha \rho_{1}+(1-\alpha) \rho_{2}$

17. $\mu_{\mathrm{m}}=\alpha \mu_{1}+(1-\alpha) \mu_{2}$

This allows construction of the RANS to be for the mixture, rather than for the individual constituent phases

18. $\frac{\partial \rho_{\mathrm{m}}}{\partial t}+\nabla \cdot\left(\rho_{\mathrm{m}} \overline{\mathbf{u}}_{\mathrm{m}}\right)=0$

$$
\rho_{\mathrm{m}}\left(\frac{\partial \overline{\mathbf{u}}_{\mathrm{m}}}{\partial t}+\overline{\mathbf{u}}_{\mathrm{m}} \cdot \nabla \overline{\mathbf{u}}_{\mathrm{m}}\right)=
$$

19. $-\nabla p+\nabla \cdot\left(\mu_{\mathrm{m}} \nabla \overline{\mathbf{u}}_{\mathrm{m}}\right)+\nabla \rho_{\mathrm{m}} \mathbf{g} \cdot \mathbf{x}-\rho_{\mathrm{m}} \nabla . \mathbf{R}+f_{\mathrm{s}}$

where $f_{\mathrm{s}}$ is the surface tension, which was formulated according to Brackbill et al. (1992).

\subsection{Boundary conditions}

Separate boundary conditions were required at the inlet, outlet and walls to represent the physical conditions. At the tank inlet, the mass flow rate was imposed by defining a fixed velocity value that acted in the normal direction to the inlet face. The turbulent quantities at the inlet were predicted from Equations 20-22 and the normal components of the Reynolds stresses were established from the turbulent kinetic energy value at the face, according to Wilcox (1994)

20. $\quad k=\frac{3}{2}\left(I_{k}|\overline{\mathbf{u}}|\right)^{2}$

21. $\varepsilon=C_{\mu} \frac{k^{3 / 2}}{l_{\mathrm{m}}}$

22. $\omega=\frac{k^{1 / 2}}{C_{\mu}^{1 / 4} l_{\mathrm{m}}}$ 


\section{Offprint provided courtesy of www.icevirtuallibrary.com Author copy for personal use, not for distribution}

The inlet eddy size $l_{\mathrm{m}}$ was selected as $10 \%$ of the inlet pipe diameter and the turbulence intensity $I_{k}$ was selected as $3 \%$. These values were not measured, but are satisfactory estimates for the inlet conditions (Versteeg and Malalasekera, 1995). This was considered reasonable, as the effects of the tank inlet conditions are somewhat isolated from the inlet of the flow control by the turbulence production and dissipation occurring in the tank volume and at the boundaries. For multi-phase simulations, the inlet fluid was set to water (i.e. a fixed value of $\alpha=1$ ).

The outlet gauge pressure was set at a fixed value of zero (i.e. atmospheric). In order to prevent non-physical results occurring in the region of the outlet due to the negative pressure gradient induced by the vortex (in the core region), a switching boundary condition was imposed on the velocity at the outlet. Where the outlet cell face velocity was out of the domain, the velocity was established by way of a zero gradient boundary condition. However, where the cell face velocity was into the domain, the velocity was evaluated by taking the explicit component of the face flux in the normal direction to the boundary, as shown by

23. $u_{\mathrm{b}}=\frac{\mathbf{S}_{\mathrm{f}}}{\left|\mathbf{S}_{\mathrm{f}}\right|} \cdot \frac{\mathbf{F}}{\left|\mathbf{S}_{\mathrm{f}}\right|}$

As the face flux is evaluated as part of the pressure-velocity coupling algorithm this ensures that the value is conservative and helps to prevent unboundedness due to regions of recirculation at the outlet. The turbulent quantities at the outlet were also managed with a switching condition based on the boundary flux. If the boundary flux was out of the domain, a zero gradient condition was applied. However, for conditions in which the flux was into the domain, boundary values for the turbulence quantities were derived in a similar fashion to the values at the tank inlet. A similar condition was also imposed on the phase fraction for multi-phase simulations. If the flux was out of the domain, the phase gradient was assumed to be zero; for cases in which the flux was into the domain, the entering fluid was assumed to be air (i.e. a fixed value of $\alpha=0$ ), which represents the free-discharge condition.

At the wall boundaries, a no-slip (fixed velocity of zero) condition was imposed on the velocity field. The turbulent behaviour was adjusted using the wall function relationship specified in Section 3.3.1. For the remaining variables at all boundaries a zero gradient condition was imposed.

\subsection{Numerical discretisation and convergence}

All of the convected quantities, with the exception of the velocities and the Reynolds stresses, were discretised using the central differencing scheme. The convective velocity terms were discretised using the second-order upwind scheme, as this was considered to describe more accurately the transportive properties of the non-linear convective terms. Second-order accurate discretisation schemes were found to produce non-physical oscilla- tions and become unbounded when applied to the convection of the Reynolds stresses, even for unsteady simulations. Benim et al. (2008) experienced similar issues and resorted to the firstorder upwind scheme, which produced results that were in close agreement with the experimental readings for a swirl combustor. Therefore, the first-order upwind scheme was selected for these terms, which was found to give satisfactory results.

The solution accuracy was controlled by setting convergence criteria for the linear solvers, and modifying the relaxation factors and time step size for steady and transient simulations, respectively. The linear solver convergence criteria for all simulations were set to reduce the absolute iteration residuals below $1 \times 10^{-6}$. After this tolerance was reached, no further iterations were performed for the transported quantity in question. When all the residuals for the transported variables fell below $1 \times 10^{-6}$, the solution was considered converged. It should also be noted that in some steady DSM cases this residual criteria was not satisfied when solving for the transport of the Reynolds stresses. A maximum residual value of $2.5 \times 10^{-2}$ was recorded for the Reynolds stresses and the residuals were typically in the region of $3 \times 10^{-3}$. It was hypothesised that the increase in residual values was caused by the DSM turbulence models resolving anisotropic eddies, which cause the vortex core to precess, resulting in quasi-steady behaviour. Due to the unsteadiness experienced with the DSM turbulence models, the final values for all steady simulations were averaged over the final 5000 iterations of the solution sequence.

An additional convergence criterion on the differential pressure across the VFC was also applied. This ensured that the pressure difference between the inlet and outlet did not vary by more than $2 \%$ over an average of the previous 5000 iterations. This was necessary, as it was found that the head loss continued to vary even when the iteration residuals were consistently below $1 \times 10^{-3}$. This measure was also helpful in determining the stability and convergence of the quasi-steady DSM and unsteady DSM simulations.

The transient simulation procedure involved obtaining a steady simulation result to provide suitable initialisation variables. The unsteady solution algorithms were then iterated over until a total of ten volume exchanges of the VFC chamber had been completed. The time step size was adjusted so that a maximum Courant number of $0 \cdot 2$ was observed in the solution domain, which was typically in the region of $2 \times 10^{-5} \mathrm{~s}$. Courant numbers above this value were found to cause the solution to become unstable and diverge. The final velocity and pressure values for the transient simulations were determined by averaging the predicted values over the final volume exchange period.

The mass continuity error between the inlet and the outlet was checked for all simulations. The maximum observed error in the flow rate for any model was calculated as $5 \times 10^{-7} \mathrm{~m}^{3} / \mathrm{s}$.

The calculations were performed on a Beowulf-style cluster at the University of Exeter. 


\section{Results}

\subsection{Sensitivity of CFD predictions to spatial discretisation}

The turbulence model selected to perform the spatial discretisation study was the DSM variant of Gibson and Launder (1978). Being the most complex of the DSM models investigated, it was considered that this model's ability to resolve anisotropic eddies in regions of varying strain rate and the inclusion of wall echo effects would make it more sensitive to the spatial discretisation than the other models investigated.

The geometry used to investigate the solution sensitivity to the spatial discretisation resolution was a VFC with an outlet diameter of $100 \mathrm{~mm}$ and a geometric swirl parameter of $3 \cdot 14$. This is the VFC geometry shown in Figure 3. The five flow rates investigated were $4,5,6,7$ and $81 / \mathrm{s}$. These flow rates were selected as they corresponded to the vortexing flow regime for this geometry, determined from experimental testing.

The simulations were performed using a steady, single-phase modelling approach. Figure 5 shows the predicted variation in head loss during the vortexing flow regime for the various discretisation resolutions. This shows that the overall head loss across the VFC was not especially sensitive to spatial discretisation, with the maximum calculated difference between the lowest and highest mesh resolutions being $+2 \cdot 8 \%$ at $61 / \mathrm{s}$. This suggests that a spatial discretisation length based on $1 / 40$ th of the chamber diameter is satisfactory, although previous numerical studies suggest that swirling flows are highly sensitive to the spatial discretisation length (Derksen, 2005; Leon et al., 2008).

To investigate further the influence of spatial discretisation resolution, pressure and velocity distributions were analysed. The averaged pressure distributions for the four discretisation resolu-

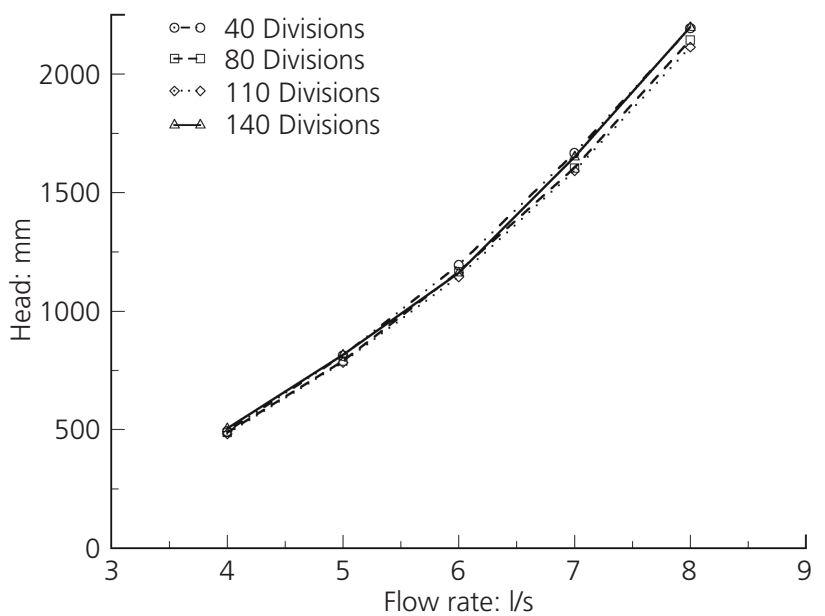

Figure 5. Hydraulic predictions from the CFD simulations for a VFC with an outlet diameter of $100 \mathrm{~mm}$ and a swirl parameter of $3 \cdot 14$ for various discretisation resolutions tions for the VFC operating at $7 \mathrm{l} / \mathrm{s}$ are displayed in Figure 6, and the vertical and axial velocity distributions are displayed in Figures 7 and 8. Although the head loss for the differing discretisation resolutions remained similar (Figure 5), the internal field values differed more noticeably. The discretisation lengths equivalent to 1/80th, 1/110th and 1/140th of the VFC chamber diameter produced similar pressure distributions (Figure 6). The root mean square (RMS) variation in the pressure distribution for the $1 / 80$ th and $1 / 110$ th division mesh was calculated as $18 \mathrm{~mm}$ and $22 \mathrm{~mm}$, respectively, when compared with the 1/140th division result. Both these values represent a variation of less than $1.3 \%$ of the overall simulated head loss. This was deemed

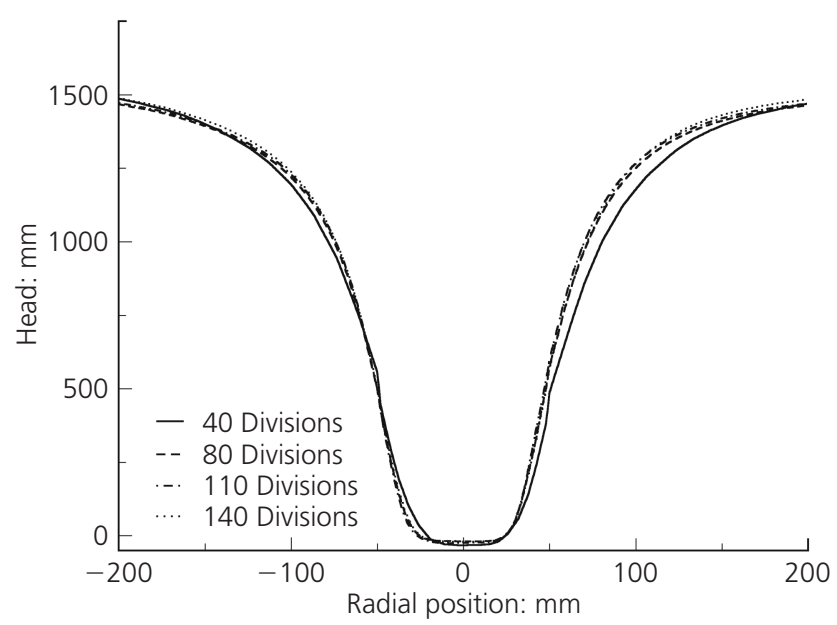

Figure 6. Predicted pressure distributions from CFD simulations across the upstream face of a VFC with an outlet diameter of $100 \mathrm{~mm}$ and a swirl parameter of 3.14 operating at $7 \mathrm{l} / \mathrm{s}$ for various discretisation resolutions

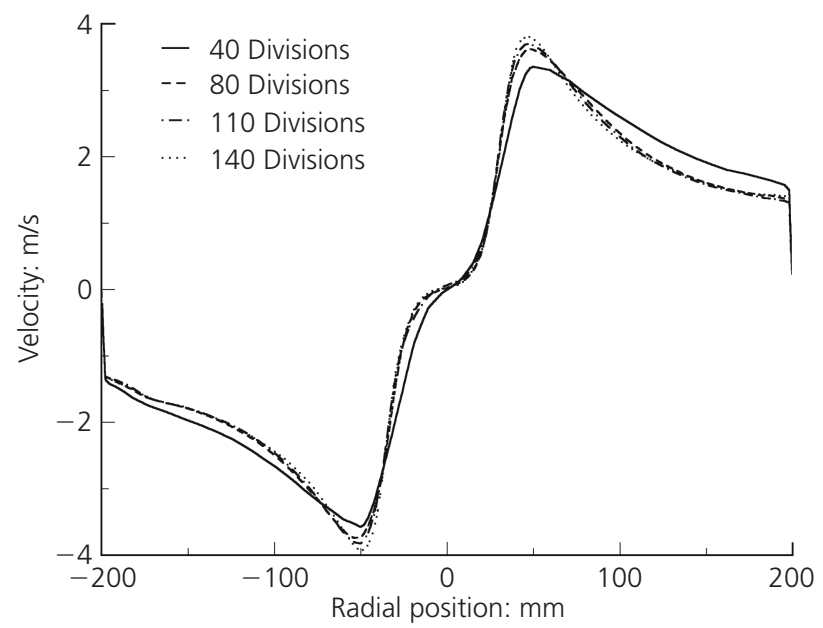

Figure 7. Predicted vertical velocity distributions across the axial mid-plane of a VFC with an outlet diameter of $100 \mathrm{~mm}$ and a swirl parameter of $3 \cdot 14$ operating at $7 \mathrm{l} / \mathrm{s}$ for various discretisation resolutions 


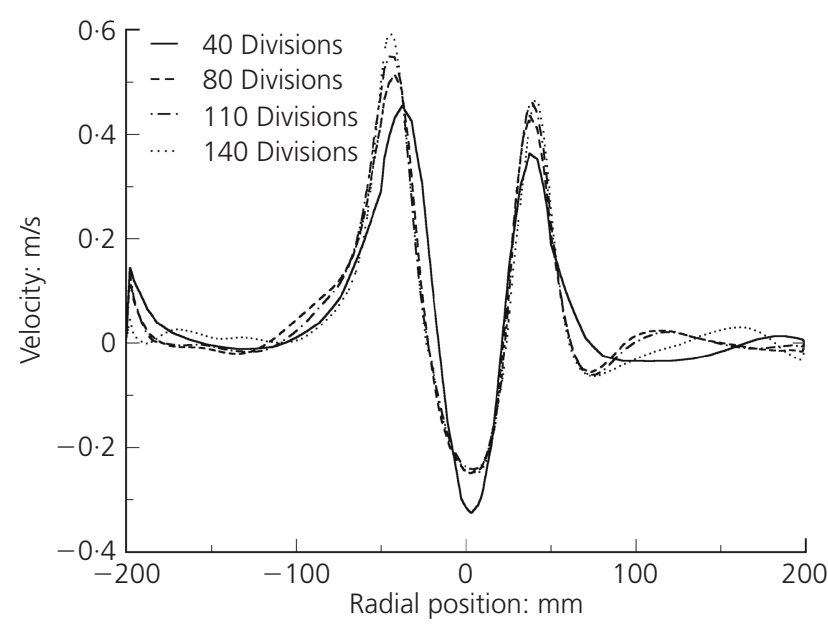

Figure 8. Predicted axial velocity distributions across the axial midplane of a VFC with an outlet diameter of $100 \mathrm{~mm}$ and a swirl parameter of 3.14 operating at $7 \mathrm{l} / \mathrm{s}$ for various discretisation resolutions

satisfactory as it was within the experimental $95 \%$ confidence interval of $1.85 \%$ for head loss. In comparison, a discretisation length of a 1/40th of the VFC chamber diameter appears to be inadequate, as the RMS variation was evaluated as $111 \mathrm{~mm}$ compared with the $1 / 140$ th mesh result. This is a variation of $6.7 \%$ of the overall head loss and is therefore outside the experimental confidence interval. A similar trend occurs for the vertical and axial velocities. From these simulations, it was determined that a discretisation length equivalent to $1 / 80$ th of the chamber diameter can be expected to deliver satisfactory predictions; as for higher mesh resolutions, the differences in pressure distributions were negligible.

Figures 7 and 8 deliver the first insights into the behaviour of the vortex in relation to the internal velocities. The vertical velocity plots (Figure 7) demonstrate that discretisation lengths equivalent to $1 / 80$ th, $1 / 110$ th and $1 / 140$ th of the chamber diameter resulted in similar distributions, whereas a discretisation length equivalent to $1 / 40$ th of the chamber diameter appeared unable to resolve the velocity gradients and the peak velocity was under-predicted compared to the other discretisation lengths. This also explains the observed difference in the pressure distribution (Figure 6) for the mesh with a discretisation length equivalent to $1 / 40$ th of the chamber diameter, as the azimuthal velocity distribution will be the dominant factor in determining the pressure distribution. The axial velocity plots shown in Figure 8 show that similar minimum velocities were predicted for meshes with discretisation lengths equivalent to $1 / 80$ th, $1 / 110$ th and $1 / 140$ th of the chamber diameter, but there was a trend of increasing maximum velocity with increasing resolution. This shows that there was still some sensitivity of the solution to the mesh, although this did not significantly affect the pressure distributions. The minimum and maximum axial velocities were approximately $25 \%$ lower for the mesh with a discretisation length of $1 / 40$ th of the chamber diameter, compared with the other mesh resolutions. Some variation is observed in the axial velocities in the vortex tail region for all of the mesh resolutions. However, as the axial velocity in this region is close to zero, the solution may be influenced by variations in mesh quality, so these fluctuations are unlikely to be significant.

Columnar vortex flows generally exhibit two distinct behaviours, which are most easily determined from the axial velocity profile as jet-like or wake-like flow distributions. Burgers (1948) presented an analytical solution to the Navier-Stokes equations that produces a jet-like axial flow profile, as the axial velocity is linearly related to the radial position. In comparison to this derivation, Sullivan (1959) formulated a solution to the NavierStokes equations that relates axial velocity to a function of the radial position. This results in a family of solutions, but the derivation most commonly associated with Sullivan is a two-celled vortex, which permits a wake-like axial velocity flow profile.

The negative axial velocities observed in Figure 8 and the inflection in azimuthal (vertical) velocity shown by Figure 7 in the core region are characteristic of the two-celled vortex distribution derived by Sullivan (1959) for confined swirling flows. This indicates that this model may be valid for predicting the velocity distributions predicted for the VFC geometries.

It should be noted that the radial distributions are not presented, as these results were of little relevance. This was due to the monitoring locations not exactly intersecting the axis of the vortex.

\subsection{Turbulence model selection}

The final turbulence model selection was determined by performing a comparison of the predictions of the five turbulence models presented in Section 3.3 against the experimental data to verify which model is most suitable. The geometry assessed was for a VFC with a $100 \mathrm{~mm}$ dia. outlet and a swirl parameter of $3 \cdot 14$ operating between $4 \mathrm{l} / \mathrm{s}$ and $81 / \mathrm{s}$. For the computational predictions, a mesh resolution of 80 divisions of the chamber radius was applied and the single-phase, steady-state solver simpleFoam was used. The comparison was performed against the surcharged assessment data. This made it directly comparable to the steady, single-phase simulation methodology.

Figure 9 shows the hydraulic predictions for the investigated turbulence models compared with the experimental VFC characteristic. The deficiencies of the Boussinesq approximationbased (EVM) models are immediately apparent, with all of the models over-predicting the head loss by more than $30 \%$. In comparison, the DSM turbulence models of Launder et al. (1975) and Gibson and Launder (1978) only varied on average by $-3 \%$ and $+0 \cdot 74 \%$, respectively.

The pressure distributions recorded across the inside of the upstream face of the VFC operating at $71 / \mathrm{s}$ are displayed against 


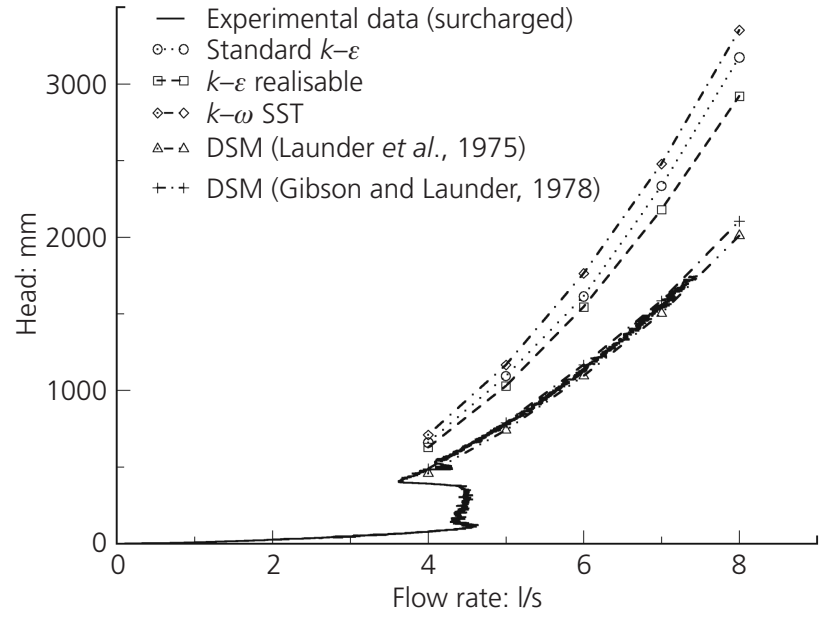

Figure 9. Comparison of the hydraulic predictions from CFD simulations for a VFC with an outlet diameter of $100 \mathrm{~mm}$ and a swirl parameter of 3.14 for various turbulence models against the experimental data

the experimental data in Figure 10. Perusal of these pressure distributions indicates that, although the EVMs are able to predict the pressure loss in the vortex core region with relative accuracy, the pressure in the vortex tail is significantly over-predicted and the correlation with the experimental data is poor. In comparison with the EVM predictions, at most monitored locations, the DSM-based turbulence models result in a strong correlation with the experimental data.

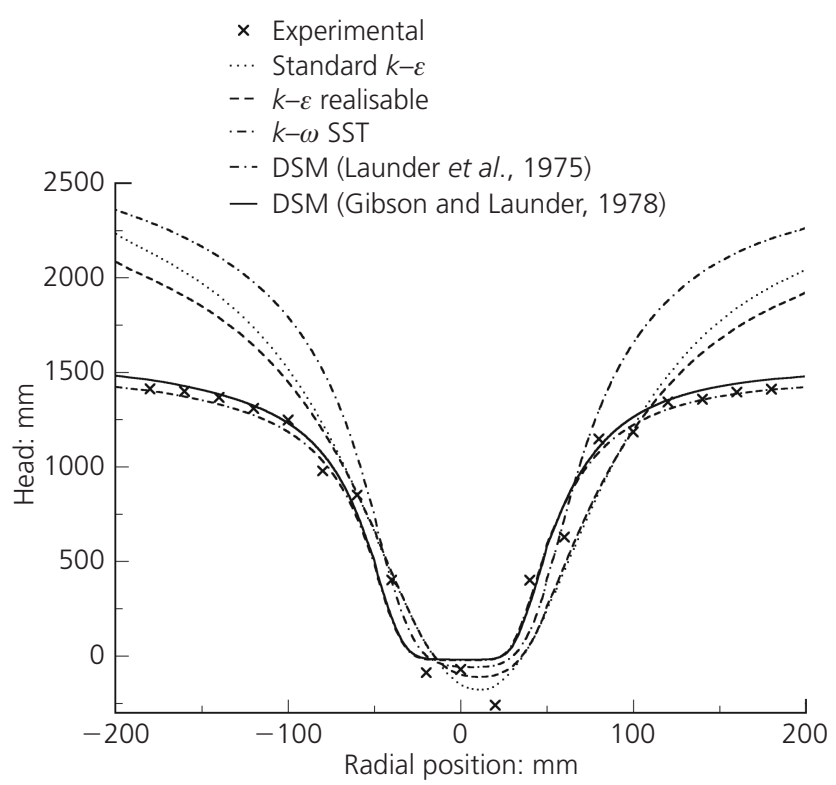

Figure 10. Predicted pressure distributions from CFD simulations across the upstream face of a VFC with an outlet diameter of $100 \mathrm{~mm}$ and a swirl parameter of 3.14 operating at $7 \mathrm{l} / \mathrm{s}$ for various turbulence models alongside the experimental values
Analysis of the vertical velocity distributions for the VFC operating at $71 / \mathrm{s}$, as shown in Figure 11, indicates that for both DSM and EVM models the peak velocities are similar, although the $k-\omega$ SST model does exhibit slightly increased values. At the chamber radius, the azimuthal velocity for all EVMs far exceeds the mean inlet velocity of $0.7 \mathrm{~m} / \mathrm{s}$, by a factor of approximately 3 , indicating a non-physical result. The $k-\omega$ SST model again shows a slight improvement in the vortex tail behaviour, but still predicts much higher velocities in this than the DSM models. This is most likely caused by the over-prediction of turbulent kinetic energy in regions of high strain rate due to the turbulent kinetic energy production term, which is proportional to the square of the strain rate

24. $\quad P_{\mathrm{k}}=2 \mu_{\mathrm{t}}|\overline{\mathrm{S}}|^{2}$

This differs from the DSM production term, which yields a linear relationship with respect to the fluid strain rate (Launder and Sandham, 2002; Revell et al., 2005) as shown by

\section{5. $\quad \mathbf{P}=-\mathbf{R} \cdot(2 \bar{S})$}

The knock-on effect of this is that EVMs exhibit an unrealistic inflation of turbulent viscosity and excessive diffusion of the velocity in the radial direction. This effectively transports the high velocities at the edge of the vortex core, across the tail region of the vortex.

Figure 12 shows the predicted turbulent viscosity distributions across the axial mid-plane of the VFC at $71 / \mathrm{s}$ for the Gibson and Launder (1978) DSM and standard $k-\varepsilon$ EVM turbulence model. This demonstrates that the peak turbulent viscosity predicted by

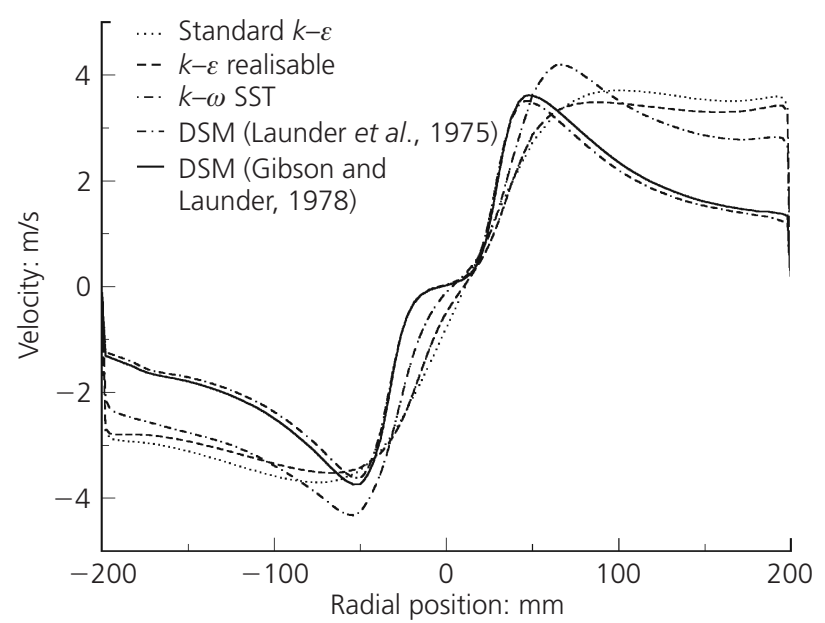

Figure 11. Predicted vertical velocity distributions from CFD simulations across the axial mid-plane of a VFC with an outlet diameter of $100 \mathrm{~mm}$ and a swirl parameter of $3 \cdot 14$ operating at $7 \mathrm{l} / \mathrm{s}$ for various turbulence models 


\section{Offprint provided courtesy of www.icevirtuallibrary.com Author copy for personal use, not for distribution}

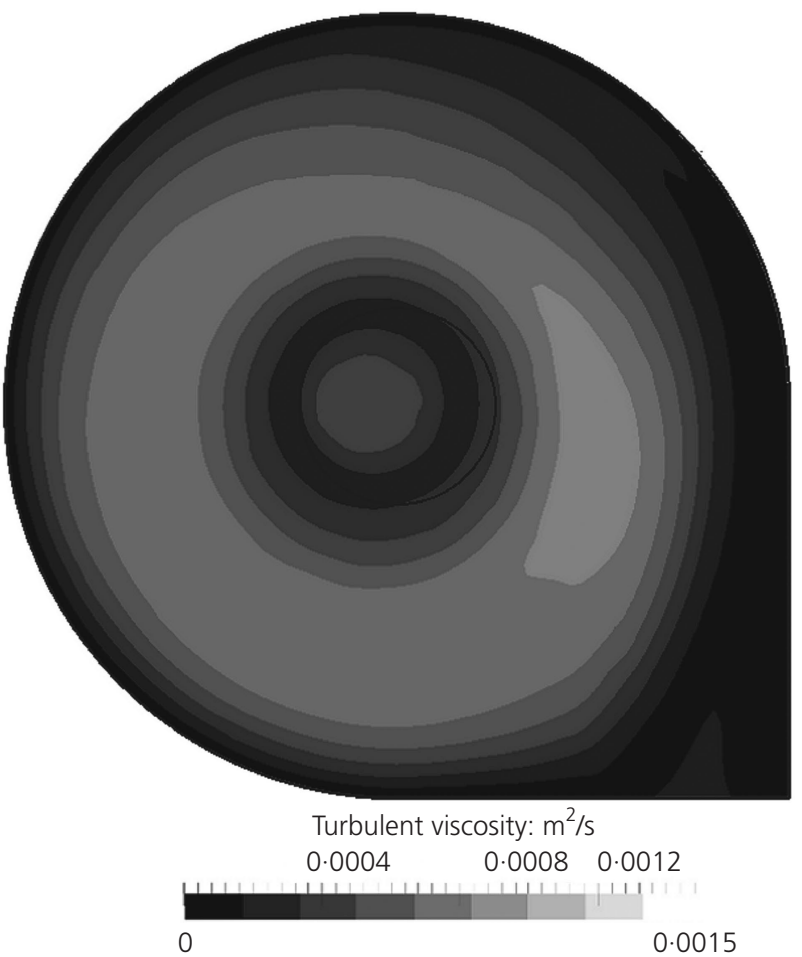

(a)

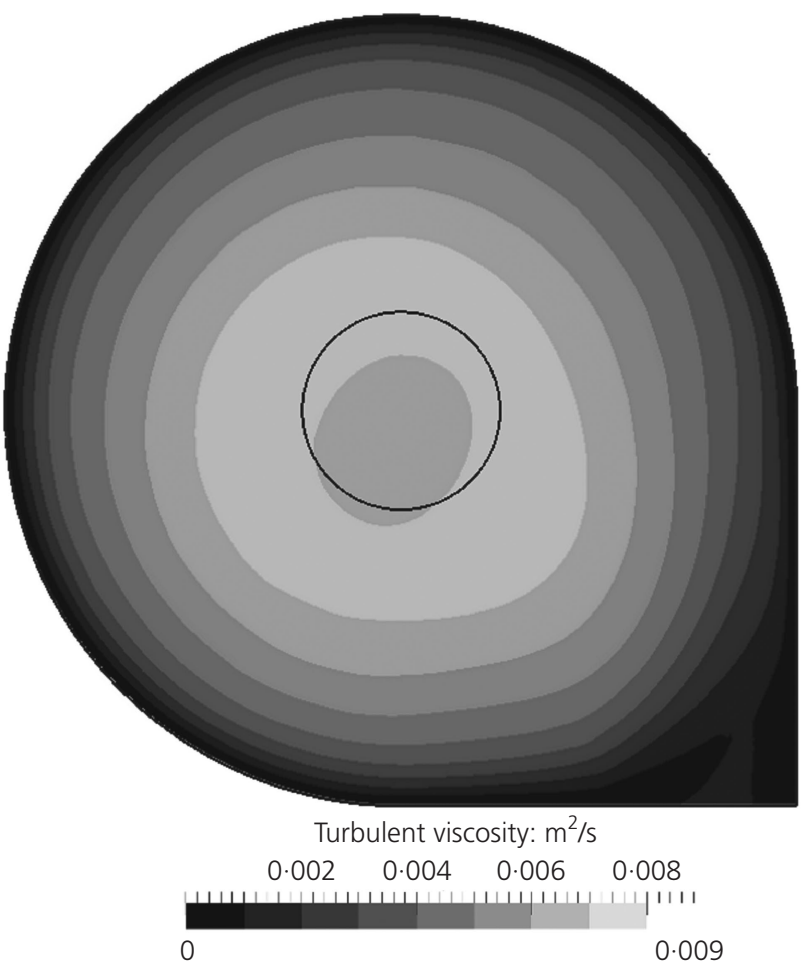

(b)

Figure 12. Predicted distributions of turbulent viscosity across the axial mid-plane of a VFC with a $100 \mathrm{~mm}$ outlet diameter and a swirl parameter of 3.14 operating at 7 I/s using: (a) Gibson and Launder (1978) DSM; (b) standard $k-\varepsilon$ EVM the Gibson and Launder (1978) DSM and standard $k-\varepsilon$ model differ by a factor of approximately 6 . Subsequently, this reinforces the hypothesis that the shortcomings of the EVM models are due to differences in turbulence production, illustrating the differences in proportionality between the production terms at work.

It is also interesting to note that the predictions of the DSM models indicate values of tangential velocity near the wall of the VFC chamber much higher than the mean inlet value of $0.7 \mathrm{~m} / \mathrm{s}$. This suggests that the peak inlet velocity, rather than the mean value, may be a more important factor in the development of vortex behaviour. Therefore, the development of the velocity profile at the inlet may have a significant effect on the confined swirling flow.

The axial velocity distributions shown in Figure 13 indicate that the standard $k-\varepsilon$ model fails to predict the negative axial velocity in the core region that is normally associated with a two-celled vortex. The realisable $k-\varepsilon$ and $k-\omega$ SST models show some improvement over the standard $k-\varepsilon$ model in that they predict negative velocities in the core region, but do not exhibit the same magnitude or symmetry predicted by the DSM models.

The differences between the predicted velocity values (Figures 11 and 13) for the DSM models are small, indicating that the influence of wall echo and blockage effects on the behaviour of VFCs is negligible. This is also confirmed by the poor correlation of the $k-\omega$ SST model, which focuses on improving near-wall behaviour, but reverts to standard $k-\varepsilon$ type behaviour in the freestream flow. As the DSM variant proposed by Gibson and Launder (1978) delivered the closest correlation to the experimentally determined pressure distribution, this turbulence model was selected as the most appropriate model for the simulation of

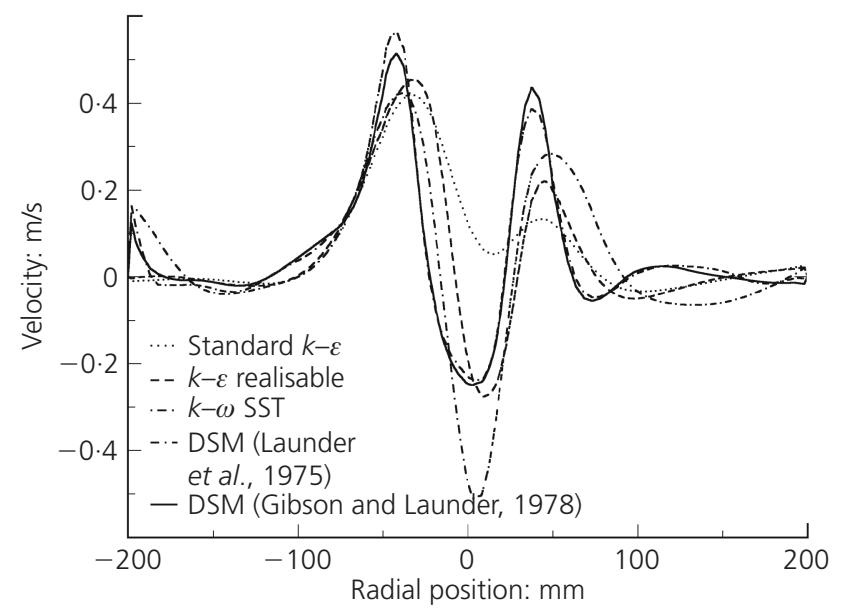

Figure 13. Predicted axial velocity distributions from CFD simulations across the axial mid-plane of a VFC with an outlet diameter of $100 \mathrm{~mm}$ and a swirl parameter of 3.14 operating at $7 \mathrm{l} / \mathrm{s}$ for various turbulence models 


\section{Offprint provided courtesy of www.icevirtuallibrary.com Author copy for personal use, not for distribution}

VFCs. The predicted velocity profiles also indicate that the vortex model proposed by Sullivan (1959) is of greatest interest for predicting the behaviour of VFCs, as it exhibits both the inflection of the tangential velocity and negative axial velocity in the vortex core region predicted by the DSM models. This is not surprising as the model proposed by Sullivan (1959) was derived specifically for the conditions of confined, turbulent, swirling flows.

\subsection{Influence of the multi-phase formulation and temporal derivatives}

In order to make predictions that more closely match the VFC free-discharge condition, a comparison between single and multiphase simulation procedures was performed. This also permitted an analysis as to whether VoF treatment of the material properties in the turbulence model was valid and able to resolve the more subtle differences in head loss associated with the introduction of air in the vortex core. For the free-discharge condition, a slight increase in the experimental head loss across the VFC is observed compared with the surcharge condition. This is most likely due to a reduction in viscous dissipation in the vortex core resulting from the presence of air rather than water. The VFC simulated had a $100 \mathrm{~mm}$ dia. outlet port and a swirl parameter of $3 \cdot 14$. Swirling flows can produce repetitive transient flow features, such as precession of the vortex core, meaning they are often classified as quasi-steady. As the multi-phase simulations were performed in an unsteady fashion, unsteady single-phase simulations were also performed to ensure that the differences between the single and multi-phase simulations were not due to the unsteady influences. All simulations in this section utilised the DSM turbulence model of Gibson and Launder (1978) as this was shown to give the closest correlation with the empirical values.

The predicted hydraulic characteristics from the single-phase, steady and unsteady predictions, and the multi-phase, unsteady simulations are displayed in Figure 14 alongside the experimental measurements. It should be noted that this figure only displays the vortexing phase of operation for the flow control. This demonstrates that the steady and unsteady single-phase models produced similar results, with both methods predicting head loss values within the experimental $95 \%$ confidence interval. The multi-phase simulation was seen to resolve the increased head loss due to the introduction of the air core, with the predictions also falling within the experimental confidence interval of the relevant experimental data. This demonstrates good correlation for both the single-phase and multi-phase simulation approaches and verifies that the interpolated values of the material properties in the VoF method give satisfactory results from the turbulence model.

A dimetric projection of a section of the simulated water surface for the VFC operating at $7 \mathrm{l} / \mathrm{s}$ and a phase fraction of 0.5 is displayed in Figure 15. The interface reconstruction scheme in interFoam appears to minimise turbulent oscillations across the phase interface at the vortex core. This is advantageous as, in other schemes, the turbulent viscosity often requires damping (intentional numerical diffusion) or spatial smoothing to prevent non-

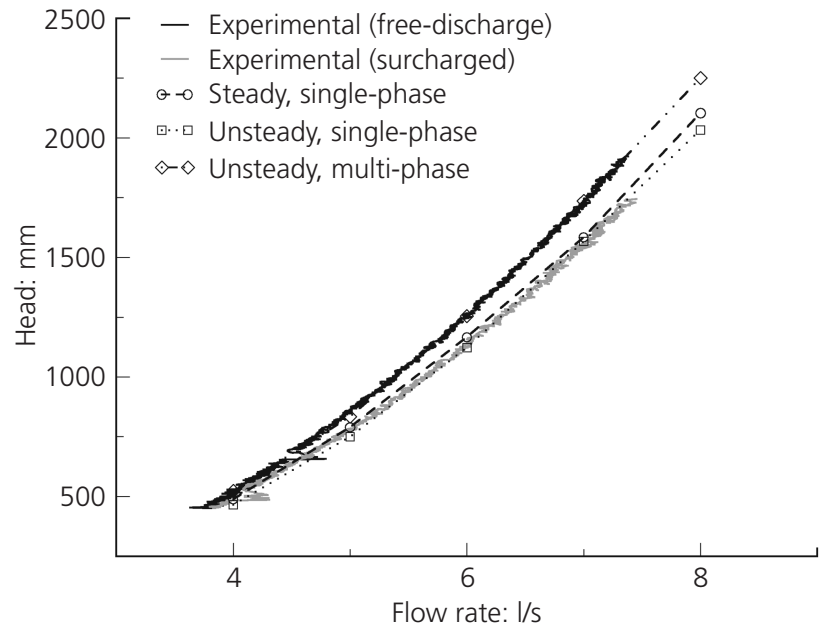

Figure 14. Comparison of single and multi-phase hydraulic predictions for a VFC with an outlet diameter of $100 \mathrm{~mm}$ and a swirl parameter of $3 \cdot 14$ plotted against the experimental head loss data

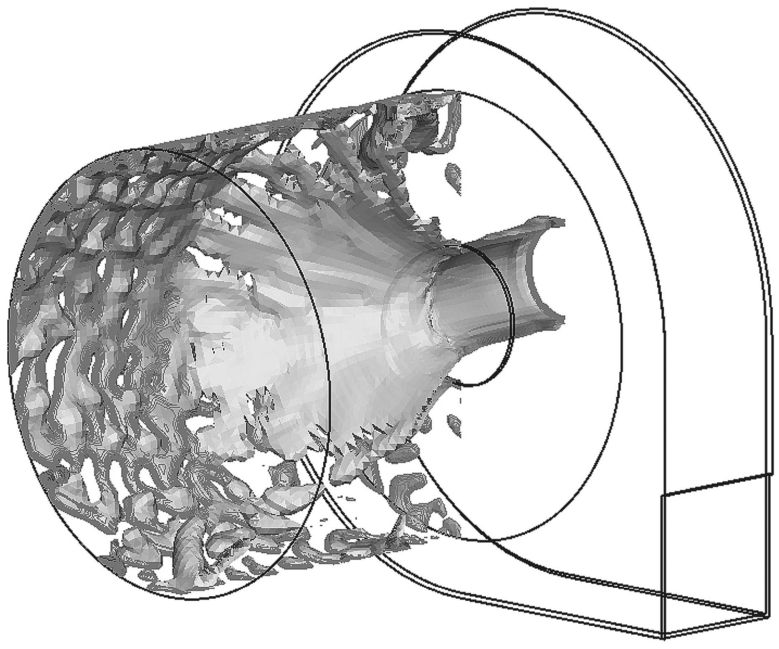

Figure 15. Dimetric projection of the 0.5 phase fraction isosurface for a VFC with an outlet diameter of $100 \mathrm{~mm}$ and a swirl parameter of $3 \cdot 14$ operating at $7 \mathrm{l} / \mathrm{s}$

physical oscillations due to the abrupt change in material properties (Prosperetti and Tryggvason, 2007). The downstream droplet breakup of the vortex fan is not well resolved, due to the highly compressive nature of the interface reconstruction scheme and inadequate spatial resolution for resolving this type of flow. These downstream influences are unlikely to affect the vortex core behaviour, which is reinforced by the correlations achieved in Figure 14.

The predicted pressure profiles across the VFC chamber from the single and multi-phase simulations for a flow rate of $71 / \mathrm{s}$ are plotted against the experimental data in Figure 16. It should be noted that the multi-phase profiles are not strictly comparable to 


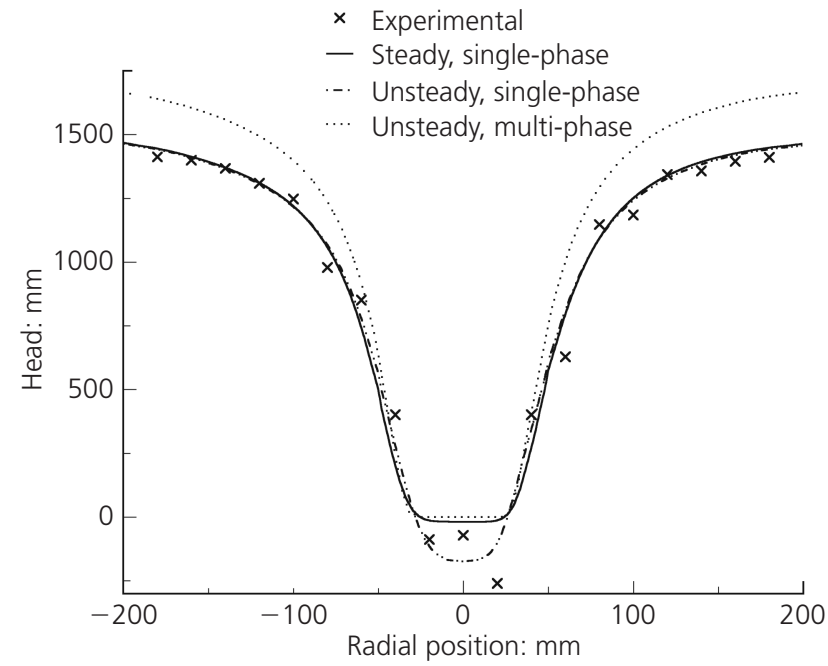

Figure 16. Single and multi-phase predictions of pressure distribution across the upstream face of a VFC with an outlet diameter of $100 \mathrm{~mm}$ and a swirl parameter of 3.14 operating at $7 \mathrm{l} / \mathrm{s}$ plotted against the experimental data

the experimental profiles, which were obtained under surcharged (single-phase) conditions, but are included as a reference. The RMS deviations of the pressure profile predictions, at flow rates of 5,6 and $7 \mathrm{l} / \mathrm{s}$, for the steady and unsteady single-phase simulations, which are comparable to the surcharged experimental assessment conditions, did not exceed $6 \%$ of the overall head loss at the simulated flow rate. The single-phase simulations show a close correlation, except in the vortex core region, where the minimum pressure predicted by the unsteady formulation is approximately $40 \mathrm{~mm}$ less than the steady value. Due to the variation in the measured values in the core region it is difficult to draw a conclusion between the validity or accuracy of the single-phase modelling methods. Both the single-phase steady and unsteady predictions show similar axial and vertical velocity profiles (Figures 17 and 18), with the unsteady formulation showing a slightly less pronounced inflection in the vertical velocity distributions and a lower minimum axial velocity in the vortex core. This may be due to the unsteady analysis method resolving the vortex core precession, whereas the steady formulation resolves the quasi-steady flow regime.

The multi-phase pressure profiles taken at 7 1/s (Figure 16) show a similar minimum pressure to the single-phase steady predictions, but a greater maximum pressure in the vortex tail. This is to be expected as the free-discharge condition resulted in a greater hydraulic loss than the surcharged results. Similar correlations were observed for the solutions produced at $5 \mathrm{l} / \mathrm{s}$ and $6 \mathrm{l} / \mathrm{s}$. The multi-phase vertical velocity profile (Figure 17) shows an increased peak velocity and an extended vortex tail. This is most likely due to the reduction in viscosity in the vortex core region, caused by the presence of air, and further explains the increased pressure loss shown in comparison with the surcharged conditions

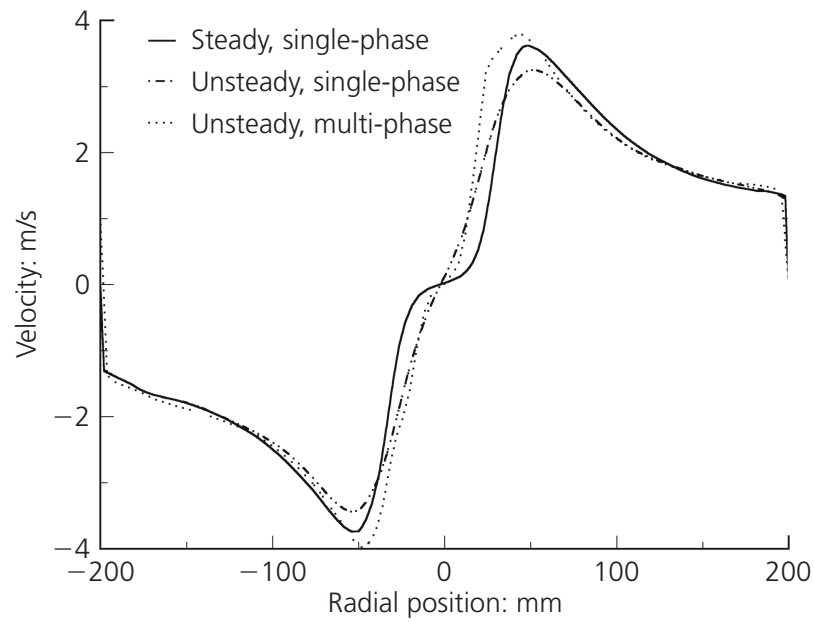

Figure 17. Single and multi-phase predictions of pressure distribution across the upstream face of a VFC with an outlet diameter of $100 \mathrm{~mm}$ and a swirl parameter of 3.14 operating at $5 \mathrm{l} / \mathrm{s}$ plotted against the experimental data

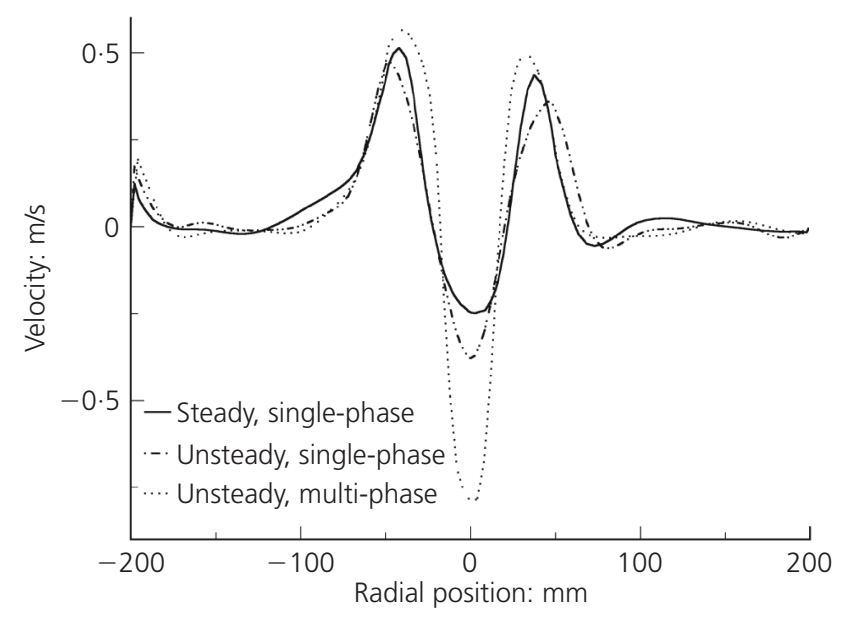

Figure 18. Single and multi-phase predictions of pressure distribution across the upstream face of a VFC with an outlet diameter of $100 \mathrm{~mm}$ and a swirl parameter of 3.14 operating at $6 \mathrm{l} / \mathrm{s}$ plotted against the experimental data

(Figure 16). Figure 18 shows that the minimum axial velocity is also significantly lower for the multi-phase formulation (indicating more pronounced flow recirculation). Again, it is probable that this is due to the reduced viscosity in the vortex core.

The correlation of the hydraulic predictions in Figure 14 was considered satisfactory evidence to verify the capability of the CFD simulation procedures in predicting the overall hydraulic behaviour and internal pressure profiles for VFCs operating under free-discharge or surcharged conditions. The assumption is also made that the satisfactory correlation in pressure distribution ensures that the prediction of the internal velocity field will be of reasonable correlation with the physical values. 


\subsection{Validity of modelling approach to other VFC geometries}

In this section, hydraulic predictions are presented for the various swirl parameters that were assessed experimentally. This aim of this action is to assess the validity of the modelling methodology for other VFC geometries. The range of geometries and flow rates simulated in order to validate the CFD simulation procedure and extend the swirl parameter range investigated experimentally is shown in Table 1. As previously stated, the flow rates investigated only cover the vortexing flow regime of operation for each VFC, not the orifice or transition flow regimes. The geometries are listed against their associated swirl parameter. All scenarios listed in Table 1 were simulated using both the steady single-phase and unsteady multi-phase methods.

For all of the simulated geometries, a small increase in Euler number was observed with increasing flow rate (Reynolds number). This trend is not examined in detail in this paper, but it is reasonable to assume that the separate scaling relationships of the losses in the near-wall region and vortex may be responsible for this variation in Euler number.

To examine the effect of swirl parameter in a more direct manner, the experimental and predicted values of Euler number were plotted against the unit swirl parameter. The results of this analysis are shown in Figure 19. All values of Euler number in Figure 19 correspond to a Reynolds number of 76000 (i.e. a flow rate of $61 / \mathrm{s}$ ) to ensure similar operating conditions, when the Reynolds number is given by

$$
\text { 26. } \operatorname{Re}=\frac{4 Q}{v \pi D_{\mathrm{o}}}
$$

The experimental readings indicate that the Euler number

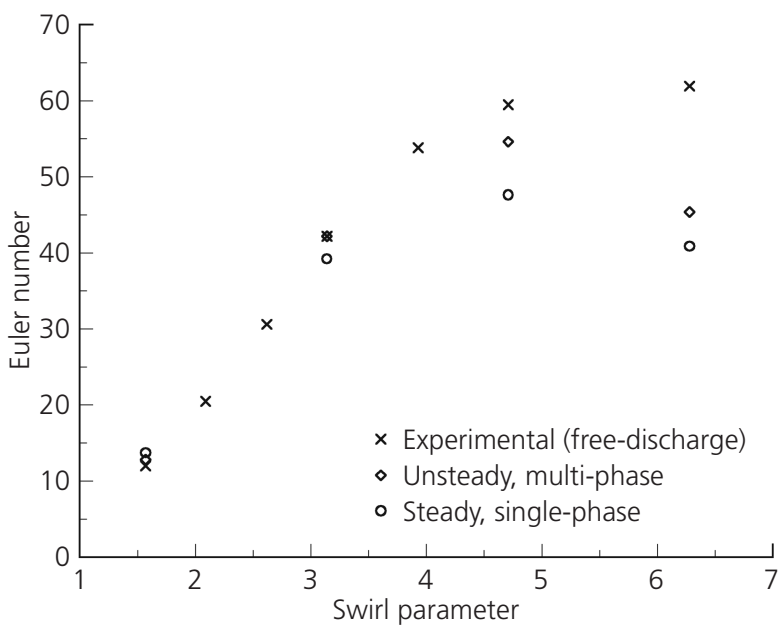

Figure 19. Variation in Euler number with swirl parameter for VFCs with an outlet diameter of $100 \mathrm{~mm}$, determined through experimental assessment and CFD simulations increases with swirl parameter and approaches a limiting value above a swirl parameter of $4 \cdot 71$, which is consistent with previous findings (Brombach, 1972; King, 1985; Priestman, 1987; Zobel, 1936). This indicates a transition away from the freevortex behaviour, in which the circulation remains constant, which is normally expected in the vortex tail region

\section{7. $\Gamma=2 \pi u_{\theta} r$}

where $\Gamma$ is the circulation value and $u_{\theta}$ is the azimuthal velocity. Figure 19 also demonstrates that the predicted values from the single and multi-phase simulations correlate well with experimental data up to a swirl parameter of $3 \cdot 14$. Above this value, both simulation methods increasingly under-predict the Euler number compared with the experimental findings. From Figure 19 it was determined that the multi-phase predictions for VFCs with swirl parameters of 1.57 and 3.14 were within the experimental uncertainty range of $1.85 \%$ of Euler number, but under-predicted the Euler number by approximately $8 \%$ for the VFC geometry with a swirl parameter of $4 \cdot 71$, increasing to an under-prediction of $27 \%$ for a swirl parameter of $6 \cdot 28$.

The maximum Euler number observed in any of the experimental assessments was 61.93 , whereas the predicted maximum for the multi-phase simulation is 54.59 , as shown in Figure 19. At a swirl parameter of $6 \cdot 28$, the single-phase and multi-phase predictions were calculated to under-predict the experimental Euler number by $26.6 \%$ and $33.8 \%$, respectively. The sources of error causing the under-predictions for the simulations were considered to be either due to a shortcoming of the turbulence model or numerical diffusion. To establish whether numerical diffusion was responsible for the under-predictions for swirl parameters above $3 \cdot 14$, the VFC geometry with a swirl parameter of $4 \cdot 71$ was simulated at $61 / \mathrm{s}$ using the finest mesh resolution. This corresponds to a discretisation length equivalent to 140 divisions of the chamber diameter. This increased the number of mesh control volumes in the modelled region from approximately 750000 to 4400000 . However, similar results were obtained at both mesh resolutions. This indicates that the under-prediction of the multi-phase simulation, compared with the experimental value, at a swirl parameter of 6.28 is unlikely to be due to numerical diffusion. Therefore, the most probable cause of the under-prediction is a shortcoming in the modelling of the turbulent quantities. The similarity of the results between the two mesh resolutions also indicates that the downstream mesh resolution (which was also refined) did not have a significant effect on the multi-phase simulations.

The vertical velocity profiles for VFCs above a swirl parameter of 3.14 suggested a gradual deviation away from the free-vortex (constant circulation) condition with increasing swirl parameter, rather than an abrupt change in behaviour. The most likely explanation for this is that the diffusive momentum terms in the Navier-Stokes equations increasingly exceed the radial pressure 


\section{Offprint provided courtesy of www.icevirtuallibrary.com Author copy for personal use, not for distribution}

gradient along the perimeter of the VFC chamber, which maintains free-vortex behaviour. To demonstrate the basis of this hypothesis, it is necessary to examine the behaviours of the radial and vertical velocities. The streamwise pressure gradient and axial velocities are neglected from this analysis as they are assumed to be negligible at a reasonable distance from the outlet. For free-vortex (potential flow) behaviour, which is expected to dominate the vortex tail region, the radial pressure gradient can be obtained from the Euler equation in cylindrical coordinates

28. $\frac{\partial p}{\partial r}=\frac{\rho U_{\theta}^{2}}{r}$

This relationship demonstrates that, for a given inlet velocity, the pressure gradient decreases linearly in relation to the VFC chamber radius. However, for a given inlet velocity, the velocity gradient along the circumference of the chamber is negligible and the diffusive effects remain constant for a given flow rate irrespective of chamber radius. Therefore, as the radius increases, the magnitude of the pressure gradient lessens relative to the diffusive terms at the circumference of the VFC chamber. When the diffusive terms approach the radial pressure gradient magnitude (Equation 29) the free-vortex motion is diffused sufficiently that constant or quiescent flow patterns dominate.

\section{9. $\left|\nabla \cdot\left[\left(\mu+\mu_{\mathrm{t}}\right) \nabla \overline{\mathbf{u}}\right]\right| /|\nabla \bar{p}| \geqslant \mid \nabla \bar{p}$}

This demonstrates that, beyond a critical radius, the free-vortex behaviour is dissipated and the vortex reverts to acting in an unconfined manner. Figure 20 shows a contour plot through the

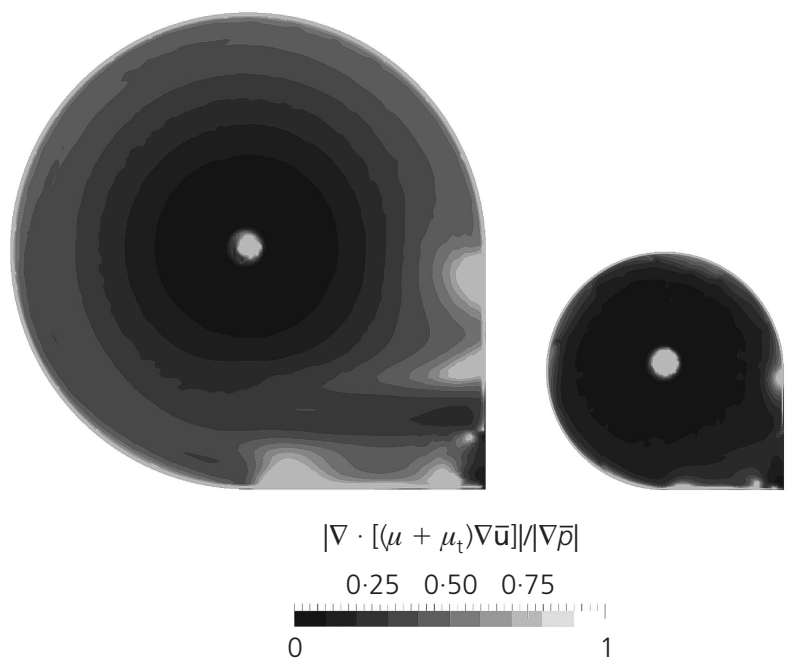

Figure 20. Comparison of regions across the axial mid-plane of a VFC with a $100 \mathrm{~mm}$ dia. outlet and swirl parameters of 6.28 (left) and $3 \cdot 14$ (right) where the diffusive or pressure gradient momentum sources are dominant axial mid-plane of the VFC geometry with swirl parameters of $6 \cdot 28$ and $3 \cdot 14$ operating at a flow rate of $7 \mathrm{l} / \mathrm{s}$. The lighter regions indicate areas where the diffusion term and the pressure gradient terms become comparable. This clearly indicates a pronounced zone along the circumference of the VFC chambers where the vortex tail is being diffused for the VFC with a swirl parameter of 6.28. Although this is only a preliminary investigation into the limitations of confined swirling flows, due to the diffusion of the vortex tail, it may be of great importance to other swirling flow devices, and indicates that further calibration of the turbulence model is required or that a different turbulence modelling approach may be more appropriate. As the diffusion in the tail region of the vortex is likely to be highly anisotropic, large eddy simulation (LES) may be a more reliable method for predicting this type of behaviour as the anisotropic eddies are resolved rather than modelled as with the RANS approach. This is therefore worthy of further analysis in the future. Moreover, this behaviour may be especially important in the numerical simulation of confined swirling flows and the development and calibration of accurate turbulence models.

Although the numerical simulations were unable to predict accurately the pressure loss for VFCs with swirl parameters larger than $3 \cdot 14$, the simulations confirm the findings of previous studies in which the maximum Euler number occurs for a swirl parameter of 4.71 and above. Beyond this swirl parameter value the free-vortex behaviour in the vortex tail region begins to be diffused. Therefore, VFC units with swirl parameters greater than 4.71 would not be advantageous. For swirl parameters below $3 \cdot 14$, the accuracy of the numerical predictions was found to give results of comparable accuracy to the experimental assessments.

\section{Conclusion}

A number of VFC geometries were simulated using single and multi-phase RANS formulations. Each element of the modelling process was examined to establish the factors with the greatest influence on the accuracy of the predictions. A mesh resolution equal to 80 divisions of the diameter of the VFC chamber was satisfactory to resolve the overall pressure gradients and azimuthal velocities. Small variations in the predicted maximum and minimum axial velocity values were evident up to 140 divisions, indicating some sensitivity in the axial velocity, although this did not affect the predicted pressure loss across the units or the internal pressure values.

Five turbulence models were assessed - three EVM models that apply the Boussinesq assumption and two DSM models. For all conditions, the EVM models over-predicted the pressure loss across the device and the internal pressure values measured across the diameter of the VFC. Analysis of the turbulent quantities indicated that this was due to over-prediction of the turbulent production quantity and diffusion of the peak azimuthal velocity (at the interface of the vortex tail and vortex core) into the vortex tail region. As a result, the EVM models were not found to be appropriate for predicting the behaviour of VFCs. 
When simulating the behaviour of VFC geometries with swirl parameters of $3 \cdot 14$ or less, the two DSM turbulence models gave predictions of pressure loss across the device that fell within the experimental confidence interval. This was true for both the single and multi-phase formulations, which demonstrated that the VoF formulation was valid and interpolating between the constituent properties of the mixture produced an appropriate behaviour in the DSM turbulence models. Also, in the swirl parameter range of less than $3 \cdot 14$, the DSM models predicted a two-cell or multi-celled vortex, where a recirculation region was present in the vortex core. A theoretical model for this type of vortex was presented by Sullivan (1959) and this may be useful in predicting internal velocities or developing a design model for VFCs.

Above a swirl parameter of $3 \cdot 14$, the DSM predictions were shown increasingly to under-predict the pressure loss across the VFCs and the resulting Euler number, with a maximum underprediction in Euler number of $27 \%$ at a swirl number of $6 \cdot 28$. This was most likely due to the diffusive terms becoming comparable to the radial pressure gradient, dissipating the freevortex behaviour tail region. This demonstrates that the DSM turbulence models require further calibration to obtain a satisfactory correlation for high swirl number devices or that another turbulence modelling approach, such as LES, may be more applicable for modelling VFCs with swirl parameters greater than $3 \cdot 14$.

The lessons learned from the investigations reported in this paper are likely to be transferable to other devices that exhibit swirling flows at high Reynolds numbers and therefore may be used to inform other CFD analysts on beneficial modelling approaches. It is intended to extend the study in the future to include LESs of the VFC geometries to confirm whether this approach can provide an improved correlation with the experimental findings for swirl parameters above $3 \cdot 14$.

\section{REFERENCES}

Andoh RYG and Declerck C (2005) A cost effective approach to stormwater management? Source control and distributed storage. Water science and Technology 36(8-9): 307-311.

Benim AC, Escudier M, Stopford PJ, Buchanan E and Syed KJ (2008) Computational investigation of turbulent swirling flows in gas turbine combustors. International Journal of Fluid Machinery and Systems 1: 1-9.

Brackbill JU, Kothe DB and Zemnach C (1992) A continuum method for modelling surface tension. Journal of Computational Physics 100(2): 335-354.

Brombach H (1972) Untersuchung stromungsmechanischer Elemente (Fluidik) und die Moglichkeit der Anwendung von Wirbelkammerelementen im Wasserbau. $\mathrm{PhD}$ thesis, Eigenverlag des Instituts fur Wasserbau der Universitat Stuttgart, Stuttgart, Germany (in German).

Burgers JM (1948) A mathematical model illustrating the theory of turbulence. Advances in Applied Mechanics 1: 197-199.

Deamer AP (1988) The Fluid Mechanics of Hydraulic Brakes for
Flood Control. Master's thesis, St. Edmund Hall, University of Oxford, Oxford, UK.

Derksen JJ (2005) Simulations of confined turbulent vortex flow. Computers \& Fluids 34(3): 301-318.

Ferziger JH and Peric M (2002) Computational Methods for Fluid Dynamics, 3rd edn. Springer, Heidelberg, Germany.

Gibson MM and Launder BE (1978) Ground effects on pressure fluctuations in the atmospheric boundary layer. Journal of Fluid Mechanics 86(3): 491-511.

Gopala V and Wachen BV (2007) Volume of fluid methods for immiscible-fluid and free surface flows. Chemical Engineering Journal 141(1-3): 204-221.

Green MJ (1988) Flow control evaluations. Proceedings of Conflo 88 - Attenuation Storage and Flow Control for Urban Catchments, Oxford, UK. Oxford University Press, Oxford, UK.

Gupta AK, Lilley DG and Syred N (1984) Swirl Flows. Abacus Press, Tunbridge Wells, UK.

Hirt CW and Nichols BD (1981) Volume of fluid (vof) method for the dynamics of free boundaries. Journal of Computational Physics 39(1): 201-225.

Hoffmann KA and Chiang ST (2000) Computational Fluid Dynamics, vol. 1, 4th edn. Engineering Education System, Wichita, KS, USA.

Jarman DS (2011) A Study of the Design of Cylindrical Vortex Flow Controls for Use in Urban Drainage Systems. $\mathrm{PhD}$ thesis, University of Exeter, Exeter, UK.

Jasak HG and Weller H (1995) Interface Tracking Capabilities of the Inter-gamma Differencing Scheme. Imperial College of Science, Technology and Medicine, London, UK, Technical report.

Jones WP and Launder BE (1972) The prediction of laminarization with a two-equation model of turbulence. International Journal of Heat and Mass Transfer 15(2): 301314.

King CF (1985) Vortex amplifier internal geometry and its effect on performance. International Journal of Heat and Fluid Flow 6(3): 160-170.

Kolmogorov AN (1942) Equations of turbulent motion of an incompressible fluid. Izvestia Academy of Science, Physics 6(1): 56-58.

Launder BE and Sandham ND (2002) Closure Strategies for Turbulent and Transitional Flows. Cambridge University Press, Cambridge, UK.

Launder BE and Sharma BI (1974) Application of the energy dissipation model of turbulence to the calculation of flow near a spinning disc. Letters in Heat and Mass Transfer 1(2): 131-138.

Launder BE and Spalding DB (1974) The numerical computation of turbulent flows. Computer Methods in Applied Mechanics and Engineering 3(2): 269-289.

Launder BE, Reece GJ and Rodi W (1975) Progress in the development of a Reynolds-stress turbulence closure. Journal of Fluid Mechanics 68(3): 537-566.

LeCornu JP and Faram MG (2006) Evolving methods for the 
calibration of flow controls for stormwater and wastewater management. Proceedings of CIWEM 4th Annual Conference, Newcastle, UK.

Leon AS, Catano-Lopera YA, Liu X, Schmidt AR and Garcia MH (2008) Experimental and CFD modeling of a vortex flow restrictor. In Proceedings of the World Environmental and Water Resources Congress 2008 (Babcock Jr RW and Walton R (eds)). ASCE, Reston, VA, USA, vol. 316, 10.1061/ 40976(316)646.

Leonard B (1988) Simple high-accuracy resolution program for convective modeling of discontinuities. International Journal for Numerical Methods in Fluids 8(10): 1291-1534.

Menter F and Esch T (2001) Elements of industrial heat transfer prediction. Proceedings of 16th Brazilian Congress of Mechanical Engineering (COBEM).

Newton CJ, Jarman DS, Memon FA, Andoh RYG and Butler D (2013) Improvements in vortex flow control design to increase sewer network flood resistance. Proceedings of 7 th International Conference on Sewer Processes and Networks, Sheffield, UK.

Parsian H and Butler D (1993) Laboratory investigation into the performance of an in-sewer vortex flow regulator. Water and Environment Journal 70(2): 182-189.

Priestman GH (1987) A study of vortex throttles. Part 1: experimental. Proceedings of the Institute of Mechanical Engineers Part C 201(5): 331-336.

Prosperetti A and Tryggvason G (2007) Computational Methods for Multiphase Flow. Cambridge University Press, Cambridge, UK.

Queguineur G, Jarman DS, Paterson E and Tabor G (2013) CFD of vortex flow controls at low flow rates. Engineering and Computational Mechanics 166(4): 211-221.
Revell AJ, Benhamadouche S, Craft T, Laurence D and Yaqobi $K$ (2005) A stress-strain lag eddy viscosity model for unsteady mean flow. In Proceedings of ERCOFTAC International Symposium on Engineering Turbulence Modelling and Measurements 6 (Rodi W (ed.)). Elsevier, Amsterdam, the Netherlands, pp. 117-126.

Schlichting $\mathrm{H}$, Gersten $\mathrm{K}$, Krause E, Oertel Jr H and Mayes C (2004) Boundary-Layer Theory, 8th edn. Springer, Heidelberg, Germany.

Shih TH, Liou WW, Shabbir A, Yang Z and Zhu J (1995) A new eddy-viscosity model for high Reynolds number turbulent flows - model development and validation. Computers \& Fluids 240(3): 227-238.

Sullivan RD (1959) A two-cell vortex solution of the NavierStokes equations. Journal of Aerospace Science 26: 767-768.

Versteeg HK and Malalasekera W (1995) An Introduction to Computational Fluid Dynamics: The Finite Volume Method. Longman, Harlow, UK.

Weller HG, Tabor G, Jasak H and Fureby C (1998) A tensorial approach to computational continuum mechanics using object-oriented techniques. Computers in Physics 120(6): 620-631.

Wilcox DC (1994) Turbulence Modelling for CFD. DWC Industries, La Cañada, CA, USA.

Wojtowicz P and Kotowski A (2009) Influence of design parameters on throttling efficiency of cylindrical and conical vortex valves. Journal of Hydraulic Research 470(5): 559565.

Zobel R (1936) Experiments on a hydraulic reversing elbow. Mitteilungen des Hydraulischen Institut der Technischen Hochschule München 8: 1-47.

\section{WHAT DO YOU THINK?}

To discuss this paper, please email up to 500 words to the editor at journals@ice.org.uk. Your contribution will be forwarded to the author(s) for a reply and, if considered appropriate by the editorial panel, will be published as a discussion in a future issue of the journal.

Proceedings journals rely entirely on contributions sent in by civil engineering professionals, academics and students. Papers should be 2000-5000 words long (briefing papers should be 1000-2000 words long), with adequate illustrations and references. You can submit your paper online via www.icevirtuallibrary.com/content/journals, where you will also find detailed author guidelines. 